\title{
High-velocity Line Forming Regions in the Type la Supernova 2009ig
}

\section{Citation}

Marion, G. H., Jozsef Vinko, J. Craig Wheeler, Ryan J. Foley, Eric Y. Hsiao, Peter J. Brown, Peter Challis, et al. 2013. "HIGH-VELOCITY LINE FORMING REGIONS IN THE TYPE la SUPERNOVA 2009ig." The Astrophysical Journal 777 (1): 40. https://doi.org/10.1088/0004-637x/777/1/40.

\section{Permanent link}

http://nrs.harvard.edu/urn-3:HUL.InstRepos:41399802

\section{Terms of Use}

This article was downloaded from Harvard University's DASH repository, and is made available under the terms and conditions applicable to Other Posted Material, as set forth at http:// nrs.harvard.edu/urn-3:HUL.InstRepos:dash.current.terms-of-use\#LAA

\section{Share Your Story}

The Harvard community has made this article openly available.

Please share how this access benefits you. Submit a story.

Accessibility 


\title{
HIGH-VELOCITY LINE FORMING REGIONS IN THE TYPE Ia SUPERNOVA 2009ig
}

\author{
G. H. Marion ${ }^{1,2}$, Jozsef Vinko ${ }^{2,3}$, J. Craig Wheeler ${ }^{2}$, Ryan J. Foley ${ }^{1,14}$, Eric Y. Hsiao ${ }^{4}$, Peter J. Brown ${ }^{5}$, Peter Challis $^{1}$, \\ Alexei V. Filippenko ${ }^{6}$, Peter Garnavich ${ }^{7}$, Robert P. Kirshner ${ }^{1}$, Wayne B. Landsman ${ }^{8}$, Jerod T. Parrent ${ }^{9}, 10$, \\ Tyler A. Pritchard ${ }^{11}$, Peter W. A. Roming ${ }^{11,12}$, Jeffrey M. Silverman ${ }^{2}$, and Xiaofeng Wang ${ }^{13,5}$ \\ ${ }^{1}$ Harvard-Smithsonian Center for Astrophysics, 60 Garden Street, Cambridge, MA 02138, USA; gmarion @ cfa.harvard.edu \\ ${ }^{2}$ University of Texas at Austin, 1 University Station C1400, Austin, TX 78712-0259, USA \\ ${ }^{3}$ Department of Optics and Quantum Electronics, University of Szeged, Dom ter 9, H-6720, Szeged, Hungary \\ ${ }^{4}$ Carnegie Observatories, Las Campanas Observatory, Colina El Pino, Casilla 601, Chile \\ ${ }^{5}$ George P. and Cynthia Woods Mitchell Institute for Fundamental Physics and Astronomy, Department of Physics and Astronomy, \\ Texas A\&M University, 4242 AMU, College Station, TX 77843, USA \\ ${ }^{6}$ Department of Astronomy, University of California, Berkeley, CA 94720-3411, USA \\ ${ }^{7}$ Department of Physics, University of Notre Dame, 225 Nieuwland Science Hall, Notre Dame, IN 46556, USA \\ ${ }^{8}$ Adnet Systems, NASA Goddard Space Flight Center, Greenbelt, MD 20771, USA \\ ${ }^{9}$ Las Cumbres Observatory Global Telescope Network, Goleta, CA 93117, USA \\ ${ }^{10}$ Department of Physics and Astronomy, Dartmouth College, Hanover, NH 03755, USA \\ ${ }^{11}$ Department of Astronomy and Astrophysics, Penn State University, 525 Davey Lab, University Park, PA 16802, USA \\ ${ }^{12}$ Space Science and Engineering Division, Southwest Research Institute, P.O. Drawer 28510, San Antonio, TX 78228-0510, USA \\ ${ }^{13}$ Physics Department and Tsinghua Center for Astrophysics (THCA), Tsinghua University, Beijing 1,00084, China \\ Received 2013 February 14; accepted 2013 September 1; published 2013 October 11
}

\begin{abstract}
We report measurements and analysis of high-velocity (HVF) (>20,000 $\mathrm{km} \mathrm{s}^{-1}$ ) and photospheric absorption features in a series of spectra of the Type Ia supernova (SN) 2009ig obtained between -14 days and +13 days with respect to the time of maximum $B$-band luminosity ( $B$-max). We identify lines of Si II, Si III, S II, Ca II, and Fe II that produce both HVF and photospheric-velocity (PVF) absorption features. SN 2009ig is unusual for the large number of lines with detectable HVF in the spectra, but the light-curve parameters correspond to a slightly overluminous but unexceptional SN Ia $\left(M_{B}=-19.46 \mathrm{mag}\right.$ and $\left.\Delta m_{15}(B)=0.90 \mathrm{mag}\right)$. Similarly, the Si II $\lambda 6355$ velocity at the time of $B$-max is greater than "normal" for an SN Ia, but it is not extreme $\left(v_{\mathrm{Si}}=13,400 \mathrm{~km} \mathrm{~s}^{-1}\right)$. The -14 days and -13 days spectra clearly resolve HVF from Si II $\lambda 6355$ as separate absorptions from a detached line forming region. At these very early phases, detached HVF are prevalent in all lines. From -12 days to -6 days, HVF and PVF are detected simultaneously, and the two line forming regions maintain a constant separation of about $8000 \mathrm{~km} \mathrm{~s}^{-1}$. After -6 days all absorption features are PVF. The observations of SN 2009ig provide a complete picture of the transition from HVF to PVF. Most SNe Ia show evidence for HVF from multiple lines in spectra obtained before -10 days, and we compare the spectra of SN 2009ig to observations of other SNe. We show that each of the unusual line profiles for Si II $\lambda 6355$ found in early-time spectra of SNe Ia correlate to a specific phase in a common development sequence from HVF to PVF.
\end{abstract}

Key words: line: formation - line: identification - supernovae: general - supernovae: individual (SN 2009ig)

Online-only material: color figures

\section{INTRODUCTION}

Research on Type Ia supernovae (SNe Ia) has been guided for many years by the general agreement that the progenitors are carbon-oxygen (C/O) white dwarf (WD) stars as first predicted by Hoyle \& Fowler (1960). This hypothesis is based on observational and theoretical evidence that thermonuclear burning of a $\mathrm{C} / \mathrm{O} \mathrm{WD}$ can produce light curves and spectra that are very similar to those observed for SNe Ia. Beyond that general statement, we lack many of the details concerning the composition of the progenitors and the physics of the explosions. In order to increase the effectiveness of SNe Ia as distance indicators we must improve our ability to predict the intrinsic brightness of individual events. Observations that provide new information about the chemical structure deepen our understanding of the explosion physics and may contribute constraints to theoretical models.

Here we identify, measure, and compare high-velocity (HVF) $\left(>20,000 \mathrm{~km} \mathrm{~s}^{-1}\right.$ ) and photospheric-velocity (PVF) absorption

\footnotetext{
${ }^{14}$ Clay Fellow, Harvard-Smithsonian Center for Astrophysics.
}

features from lines of Si II, Si III, S II, Ca II, and Fe II in a sequence of optical spectra of SN Ia 2009ig. The spectra of SN 2009ig at phases earlier than +3 days were previously published by Foley et al. (2012). The phase of each observation is expressed in days with respect to the time of $B$-band maximum light, $B$-max (September 6.0 UT = JD 2,455,080.5; see Section 2.1).

High-velocity (HVF) absorption features from Ca II lines are frequently identified in spectra of $\mathrm{SNe}$ Ia obtained a week or more before $B$-max, but reliable identifications of HVF from other elements are rare. The presence of HVF from other lines is inferred from unusual PVF line profiles in very early-time spectra. HVF components of blended Si II $\lambda 6355$ features are reported by Mattila et al. (2005), Quimby et al. (2006), Stanishev et al. (2007), Garavini et al. (2007), Tanaka et al. (2008), and Wang et al. (2009b). Evidence for HVF in Fe II features is discussed by Branch et al. (2003), Branch et al. (2005), Branch et al. (2007), Hatano et al. (1999), and Mazzali et al. (2005a).

The two earliest spectra of SN 2009ig ( -14.5 days, -13.5 days) clearly resolve HVF Si II $\lambda 6355$ as a separate absorption feature produced in a detached line forming region. All features identified in the earliest spectra of SN 2009ig are HVF. 
The first unambiguous detection of PVF occurs at -12 days, and we measure both HVF and PVF velocities from -12 days until HVF are no longer detectable at about -6 days. All absorption features in spectra obtained after -5 days are PVF. The early start and dense coverage of our sample allows us to map a complete transition from HVF to PVF in SN 2009ig.

The strongest absorption features in early-time spectra of SN Ia are the CaII $\mathrm{H} \& \mathrm{~K}$ lines and the CaII near-infrared triplet (IR3). Most of the pre-maximum spectra in our SN 2009ig sample include both of these lines, presenting an opportunity to study the development of HVF and PVF in Ca II. Wang et al. (2006) and Patat et al. (2009) also reported simultaneous observations of HVF and PVF from both Ca II lines.

HVF from Ca II are usually observed as very broad features that extend 15,000-25,000 $\mathrm{km} \mathrm{s}^{-1}$ beyond typical PVFs. They are most often detected in spectra obtained earlier than -5 days, but it is not unusual for Ca II HVF to persist as late as maximum light. Detections of HVF Ca II in early spectra of SNe Ia are reported and discussed by Fisher et al. (1997), Hatano et al. (1999), Wang et al. (2003), Gerardy et al. (2004), Thomas et al. (2004), Branch et al. (2008), Tanaka et al. (2008), Branch et al. (2009), Marion et al. (2009), and others. Childress et al. (2013a) compare and discuss Ca II HVF in SNe Ia observed near $B$-max. Mazzali et al. (2005a) assert that all spectra of SNe Ia observed more than one week before maximum will exhibit HVF Ca II.

Foley et al. (2012) use the same pre-maximum data of SN 2009ig as we do to identify and discuss differences in line profiles of Ca II H\&K, CaII IR3, and Si II $\lambda 6355$. They reveal the two-component nature of early Si II $\lambda 6355$ features and propose that HVF may be ubiquitous in SN Ia. Here we focus on the location and composition of HVF line forming regions. In particular, we examine the characteristics of HVF from lines that are not Ca II. We directly compare the development of Si II $\lambda 6355$ features in the spectra of SN 2009ig to sequences of early-time spectra of five other SN Ia.

Velocity measurements show that the HVF and PVF line forming regions remain separated by about $8000 \mathrm{~km} \mathrm{~s}^{-1}$ in SN 2009ig. The expansion of SNe Ia is assumed to be homologous, so that measured velocities are proportional to distances from the center of the SN. Consequently, a line forming region that produces HVF absorption features is physically outside of a region that produces lower velocity features. Homologous expansion ensures that they will remain in the same relative positions.

The formation of detached HVF requires a region with a localized enhancement of abundance or density. The HVF layer must also be farther from the center of the SN than the photospheric layer. We are a long way from a consensus for how HVF line forming regions come into existence. A few of the proposed explanations for HVF are uneven density profiles in the ejecta (Mazzali et al. 2005a), a clumpy structure or a thick torus (Tanaka et al. 2006), and interaction between the SN ejecta and surrounding material (Gerardy et al. 2004). Evidence for polarization of the HVF in spectra of SNe Ia was first presented by Wang et al. (2003) and discussed by Kasen et al. (2003). If the HVF regions are not spherically symmetric, then viewing angles will influence the observed velocities.

In Section 2 we describe the observations of SN 2009ig and the reduction of the data. A discussion of the methods used for identification and measurement of absorption features is presented in Section 3. The characteristics of HVF from specific lines are presented in Section 4. A discussion of PVF and their
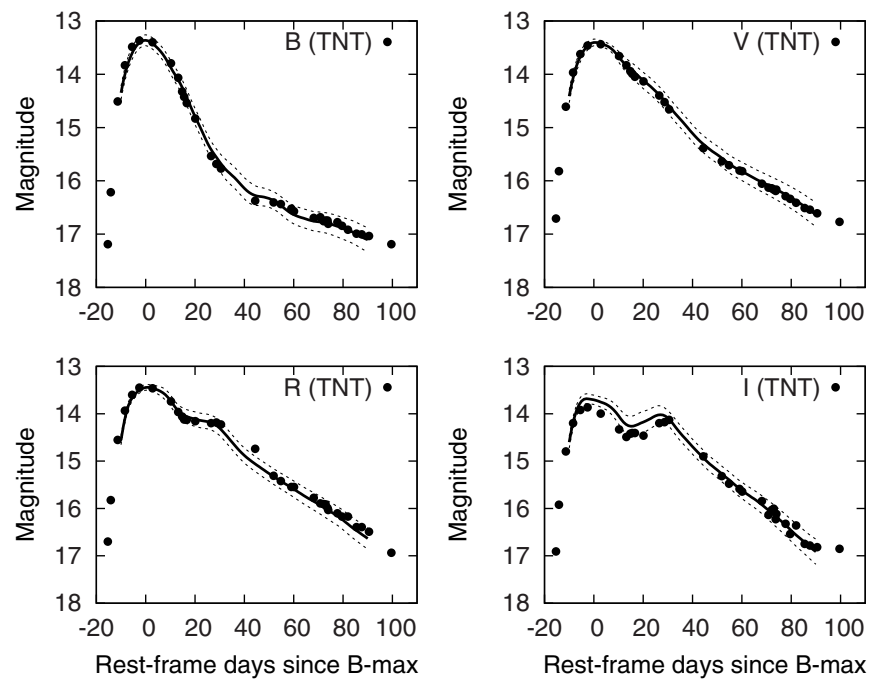

Figure 1. BVRI light curves of SN 2009ig obtained at the Tsinghua-NAOC Telescope (TNT). The black solid line is the MLCS2k2 fit and the dotted lines are the $1 \sigma$ boundaries. Template fitting gives $M_{B}=-19.5 \mathrm{mag}$ on 2009 September 6.0 with $\Delta m_{15}(B)=0.90 \mathrm{mag}$.

measurements appears in Section 5. Analysis of the composition and location of HVF line forming regions is found in Section 6. In Section 7 we compare the results for SN 2009ig to early-time spectra of other SNe Ia. Possible clues to the origins of HVF regions in $\mathrm{SNe}$ Ia are discussed in Section 8. Section 9 presents a summary and conclusions.

\section{OBSERVATIONS}

SN 2009ig in NGC 1015 (redshift $z=0.008770$; NED) was discovered (Kleiser et al. 2009) by the Lick Observatory Supernova Search (LOSS; Filippenko 2001) with the $0.76 \mathrm{~m}$ Katzman Automatic Imaging Telescope (KAIT) at a magnitude of 17.5 on 2009 August 20.5 (UT dates are used throughout this paper). The last known nondetection was also by LOSS/KAIT on August 16 to a limiting magnitude of 18.7. On August 21.1 the first spectrum was obtained with the Asiago $1.82 \mathrm{~m}$ telescope by Navasardyan et al. (2009). It revealed characteristics of an SN Ia very soon after the explosion with a high expansion velocity for Si II $\lambda 6355\left(24,500 \mathrm{~km} \mathrm{~s}^{-1}\right)$ and no detection of Si II $\lambda 5971$ or S II $\lambda 5641$. Because SN 2009ig was nearby and the detection was very early, we were presented with a unique opportunity to conduct extensive observations of a young SNe Ia.

\subsection{Photometry}

Photometric data were obtained in the $U B V I$ bands with the $0.8 \mathrm{~m}$ Tsinghua-NAOC Telescope (TNT), located at Xinglong Station of the National Astronomical Observatory of China, $180 \mathrm{~km}$ from Beijing. Details of the TNT, detectors, and observing conditions are described by Wang et al. (2008). All photometric data were reduced using standard IRAF packages.

The light curves were fit to MLCS2k2 templates (Jha et al. 2007). The light curves and the best-fit templates are displayed in Figure 1. We adopted $A_{V}(\mathrm{Gal})=0.089 \mathrm{mag}$ for the Milky Way extinction (Schlafly \& Finkbeiner 2011) and a time of maximum $B$-band brightness of $T\left(B_{\max }\right)=55080.50 \mathrm{MJD}$ (2009 September 6.0). MLCS2k2 finds $\Delta=-0.24 \pm 0.08$ for the best-fit light-curve parameter, $\mu_{0}=32.82 \pm 0.09$ mag for the distance modulus (assuming $H_{0}=73 \mathrm{~km} \mathrm{~s}^{-1} \mathrm{Mpc}^{-1}$ ), and $A_{V}$ (host) $=0.01 \pm 0.01 \mathrm{mag}$ for the host-galaxy extinction. 
From the MLCS2k2 results we find $M_{B}=-19.46 \pm 0.12$ and $M_{V}=-19.42 \pm 0.12 \mathrm{mag}$ on 2009 Sepember 6.0. The $\Delta=-0.24$ parameter corresponds to a decline-rate value of $\Delta m_{15}(B)=0.90 \pm 0.07 \mathrm{mag}$. These values suggest that $\mathrm{SN}$ 2009 ig is slightly more luminous and has a slower decline rate than a "normal" SN Ia, but the $M_{B}$ and $\Delta m_{15}(B)$ values are not exceptional.

These parameters are consistent with those of Foley et al. (2012), who used KAIT and Swift photometry of SN 2009ig to derive values of $\mu_{0}=32.96 \pm 0.02 \mathrm{mag}$ and $A_{V}$ (host) $=0.01 \pm$ $0.01 \mathrm{mag}$. Small differences in the photometric parameters have no effect on the spectroscopic results presented in this paper.

\subsection{Spectroscopy}

Our complete sample includes 32 optical spectra of SN 2009ig obtained from -14.5 days to +12.5 days with respect to the time of $B$-max. Spectra obtained before Sepember 9 ( +3 days) were previously published by Foley et al. (2012). In most cases where the phase is discussed in the text, we round the value to whole days for simplicity. The Swift $U$-grism spectra are included in this list of "optical" spectra since we only display and analyze the portion of these spectra in the range 3200-4400.

Figure 2 displays spectra obtained on 25 of the 28 nights between -14 days and +13 days. The maximum wavelength range of 3200-9000 Å reaches both the Ca II H\&K lines ( $\lambda 3945)$ and the Ca II IR3 ( $\lambda 8579)$. Full wavelength coverage is achieved on every night from -14 days to -5 days.

For some of the dates, observations of SN 2009ig that cover slightly different wavelengths occurred at two different telescopes within a few hours of each other. We combine these data to create a single continuous spectrum by trimming the data from one source at a specific wavelength and continuing from that wavelength with data from the other source without overlap. The transition points are selected to optimize the signalto-noise ratio for the combined spectrum. Spectral features in SNe Ia change rapidly at early times, but a daily cadence is sufficient to trace the development of individual features. The use of a single spectrum per day makes it easier to measure and display the data. A complete list of the observational details can be found in Table 1, including the wavelengths at which the spectra were joined.

For the ground-based spectroscopy, the slit was generally oriented along the parallactic angle to minimize differential slit losses caused by atmospheric dispersion (Filippenko 1982). SN 2009ig was observed using the Shane $3 \mathrm{~m}$ telescope at Lick Observatory with the Kast spectrograph (Miller \& Stone 1993) on August 22, 24, 25, 27, and 28. An additional spectrum was obtained on August 22 at the $10 \mathrm{~m} \mathrm{Keck}$ I telescope with the Low Resolution Imaging Spectrometer (LRIS; Oke et al. $1995)$ equipped with an atmospheric dispersion corrector. The Lick/Kast spectra used a 600/4310 grism on the blue side and a 300/7500 grating on the red side with a $2^{\prime \prime}$ wide slit. The Keck/LRIS spectrum was obtained with a 400/3400 grism on the blue side and a 600/7500 grating on the red side using a $1^{\prime \prime}$ wide slit.

The 9.2 m Hobby-Eberly Telescope (HET; Ramsey et al. 1998) with the Marcario Low-Resolution Spectrograph (LRS; Hill et al. 1998) was used to observe SN 2009ig on August 23 (-13 days). Additional HET observations were made on August 29, 30, and 31, as well as on September 2, 3, 4, 5, 9, 10, and 14. The HET/LRS spectra have an effective wavelength range of 4400-9200 А. Reduction of the HET/LRS data was also conducted with standard IRAF procedures.

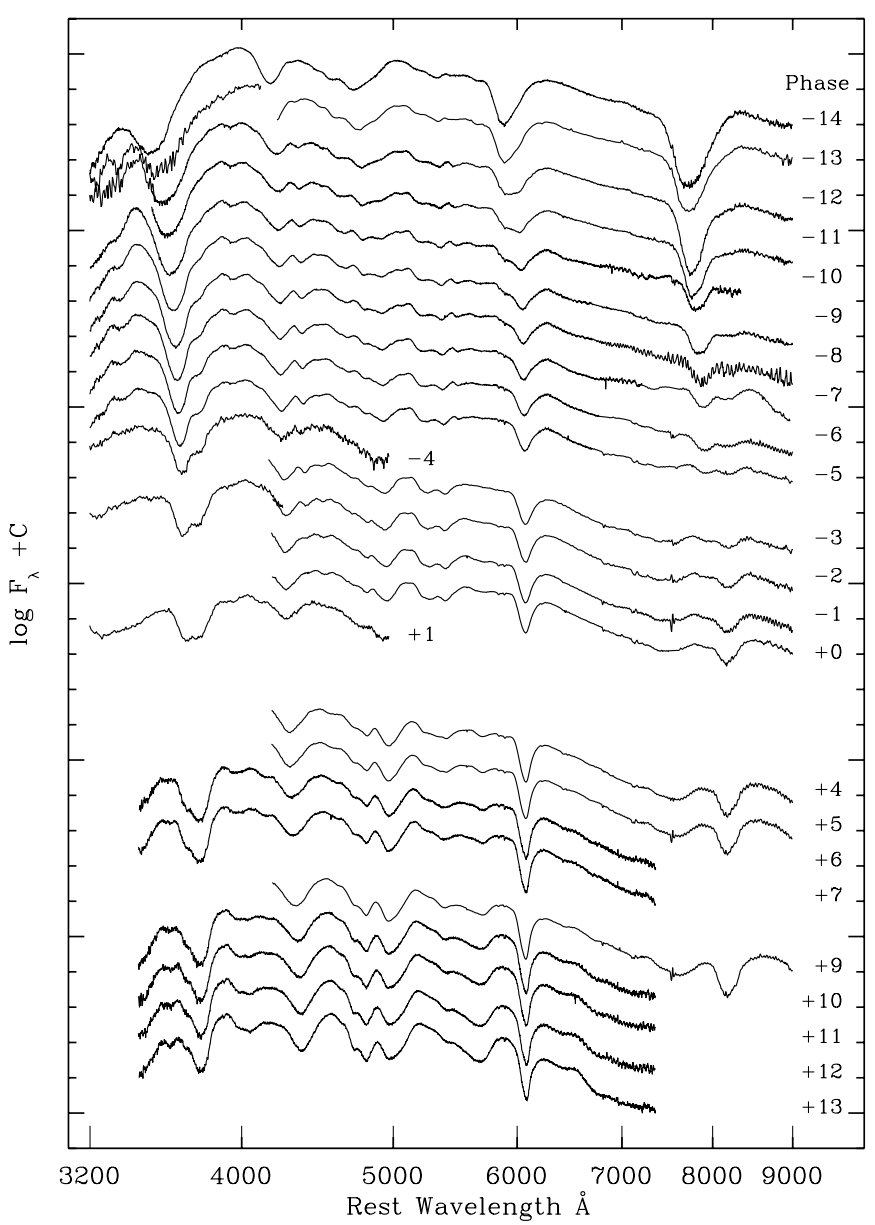

Figure 2. Spectra of SN 2009ig obtained between -14 days (top) and +13 days (bottom) with respect to the time of $B$-max (2009 Spetember 6.0). The spectra are positioned in the figure to align the continuum near $4700 \AA$ with the phase as indicated on the ordinate. CCD fringing is apparent at the red end in a few of the spectra. Ca II features are strong near $3800 \AA$ for $H \& \mathrm{~K}$ and near $8000 \AA$ for IR3, while Si II $\lambda 6355$ is prominent near $5900 \AA$. HVF components dominate the earliest spectra. There is a period of transition when both HVF and PVF can be detected from the same lines, and most lines are exclusively PVF after -6 days. Full wavelength coverage is achieved for some of the dates by combining spectra. See Table 1 for details of the observations and Section 2.2 for an explanation of how the spectra were merged.

Low-dispersion spectra of SN 2009ig were obtained on August 26, 27, 28, 29, and 30 using the $6.5 \mathrm{~m}$ MMT with the Blue Channel spectrograph (Schmidt et al. 1989), covering the wavelength range $3200-8200 \AA$. CCD processing and spectrum extraction for these observations were completed with IRAF and the data were extracted with the optimal algorithm of Horne (1986). After the wavelength calibration was derived from loworder polynomial fits to calibration-lamp spectra, additional small adjustments were applied by cross-correlating a template sky spectrum to the night-sky lines that were extracted along with the SN. IDL routines were used to flux calibrate the data and to remove telluric lines (Wade \& Horne 1988; Matheson et al. 2000; Foley et al. 2003).

The Swift satellite observed SN 2009ig with the UV grism mode of the Ultraviolet/Optical Telescope (Roming et al. 2005) onboard the spacecraft (Gehrels et al. 2004). Swift data were obtained on August 23, 25, and 27, and on September 1, 3, and 6. We do not use all of the Swift data for our analysis, but the complete sequence of Swift spectra and photometric measurements was published by Foley et al. (2012). Swift 
Table 1

Spectroscopic Observations

\begin{tabular}{lcccc}
\hline \hline Date & Phase $^{\mathrm{a}}$ & Telescope/Instrument & $\begin{array}{c}\text { Range }^{\mathrm{b}} \\
(\AA)\end{array}$ & $\begin{array}{c}\lambda \text { Merge }^{\mathrm{c}} \\
(\AA)\end{array}$ \\
\hline Aug 22.5 & -14.5 & Lick/Kast & $3400-9000$ & 6700 \\
Aug 22.6 & -14.4 & Keck I/LRIS & $3100-7860$ & 6700 \\
Aug 23.4 & -13.6 & HET/LRS & $4210-9000$ & 4225 \\
Aug 23.6 & -13.4 & Swift/Ugrism & $3200-4100$ & 4070 \\
Aug 24.5 & -12.5 & Lick/Kast & $3500-9000$ & $\ldots$ \\
Aug 25.5 & -11.5 & Lick/Kast & $3500-9000$ & 4000 \\
Aug 25.6 & -11.4 & Swift/Ugrism & $3200-4400$ & 4000 \\
Aug 26.5 & -10.5 & MMT/Blue Channel & $3190-8330$ & $\ldots$ \\
Aug 27.5 & -9.5 & Lick/Kast & $3400-9000$ & 6700 \\
Aug 27.5 & -9.5 & MMT/Blue Channel & $3180-8270$ & 6700 \\
Aug 28.5 & -8.5 & Lick/Kast & $3400-9000$ & 6700 \\
Aug 28.5 & -8.5 & MMT/Blue Channel & $3180-8320$ & 6700 \\
Aug 29.4 & -7.6 & HET/LRS & $7170-9000$ & 7210 \\
Aug 29.5 & -7.5 & MMT/Blue Channel & $3190-8270$ & 7210 \\
Aug 30.4 & -6.6 & HET/LRS & $4710-9000$ & 6850 \\
Aug 30.4 & -6.6 & MMT/Blue channel & $3180-8250$ & 6850 \\
Aug 31.4 & -5.6 & HET/LRS & $4170-9000$ & $\ldots$ \\
Sep 1.6 & -4.4 & Swift/Ugrism & $3200-4900$ & $\ldots$ \\
Sep 2.4 & -3.6 & HET/LRS & $4160-9000$ & $\ldots$ \\
Sep 3.6 & -2.4 & Swift/Ugrism & $3200-4400$ & 4200 \\
Sep 3.5 & -2.5 & HET/LRS & $4190-9000$ & 4200 \\
Sep 4.4 & -1.6 & HET/LRS & $4180-9000$ & $\ldots$ \\
Sep 5.4 & -0.6 & HET/LRS & $4180-9000$ & $\ldots$ \\
Sep 6.7 & +0.7 & Swift/Ugrism & $3200-4900$ & $\ldots$ \\
Sep 9.4 & +3.4 & HET/LRS & $4180-9000$ & $\ldots$ \\
Sep 10.4 & +4.4 & HET/LRS & $4180-9000$ & $\ldots$ \\
Sep 11.5 & +5.5 & FLWO/FAST & $3300-7200$ & $\ldots$ \\
Sep 12.5 & +6.5 & FLWO/FAST & $3300-7200$ & $\ldots$ \\
Sep 14.4 & +8.4 & HET/LRS & $4180-9000$ & $\ldots$ \\
Sep 15.5 & +9.5 & FLWO/FAST & $3300-7200$ & $\ldots$ \\
Sep 16.5 & +10.5 & FLWO/FAST & $3300-7200$ & $\ldots$ \\
Sep 17.4 & +11.5 & FLWO/FAST & $3300-7200$ & $\ldots$ \\
Sep 18.5 & +12.5 & FLWO/FAST & $3300-7200$ & $\ldots$ \\
\hline & & & & \\
\hline
\end{tabular}

Notes. The spectra obtained before September 9 were previously published by Foley et al. (2012).

${ }^{\text {a }}$ Phase in days with respect to the time of $B$-max (UT 2009 September $6.0=$ JD 2,455,080.5).

${ }^{b}$ Wavelength range used for this paper. The complete spectra may cover a larger range.

c Wavelength at which this spectrum was trimmed and combined with a contemporaneous spectrum to form a single spectrum for this date.

data were extracted and reduced using the "uvotimgrism" package of FTOOLS. The effective wavelength coverage is $1700-4900 \AA$, but in this analysis we use only a small portion of the Swift spectra covering the wavelength region 3200-4400 ̊ that contains the $\mathrm{Ca}$ II $\mathrm{H} \& \mathrm{~K}$ feature.

\section{LINE IDENTIFICATIONS AND VELOCITY MEASUREMENTS}

Eight lines have been identified that produce both $\mathrm{HVF}$ and PVF absorptions in spectra of SN 2009ig. The features associated with these lines are displayed by phase and velocity space in four figures: Si III $\lambda 4560$ and Si II $\lambda 6355$ (Figure 3), S II $\lambda \lambda$ 5453, 5641 (Figure 4), Ca II H\&K $\lambda 3945$ and Ca II IR3 $\lambda 8579$ (Figure 5), and Fe II $\lambda \lambda$ 5018, 5169 (Figure 6). The velocity measurements at each phase are indicated by a blue hashmark for HVF and a red hashmark for PVF. To facilitate comparison of the components between features, all of the figures also have a dotted line plotted at $21,500 \mathrm{~km} \mathrm{~s}^{-1}$ and a dashed line at $13,000 \mathrm{~km} \mathrm{~s}^{-1}$. These velocities were selected

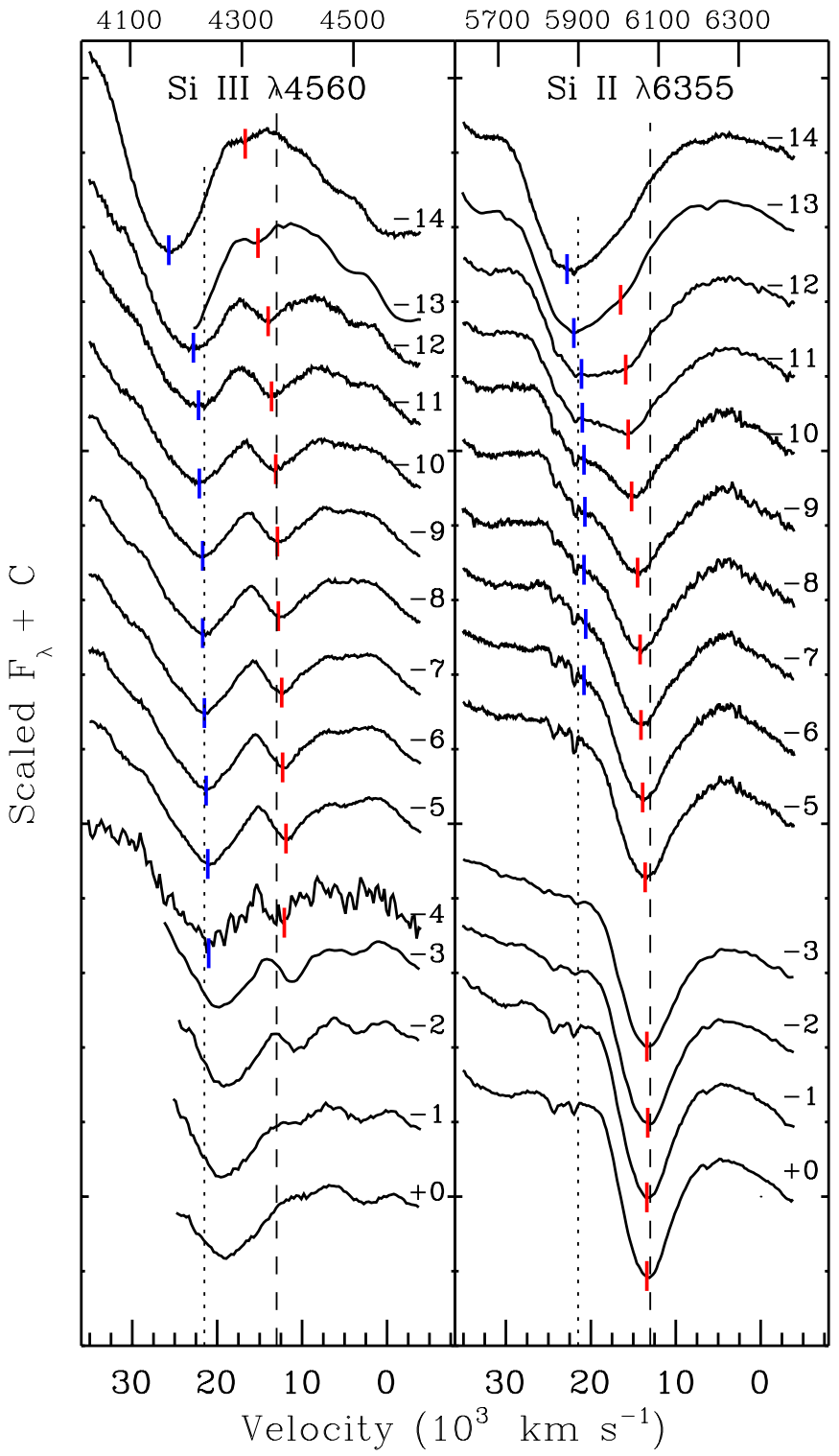

Figure 3. HVF and PVF for Si III $\lambda 4560$ (left) and Si II $\lambda 6355$ (right) in SN 2009 ig are displayed in velocity space (blueshifted velocities are expressed with positive values). Rest wavelengths are indicated on the top abscissa. The spectra were obtained between -14 days and $B$-max, and the phase of each spectrum is marked near the red end. The line profiles for Si II $\lambda 6355$ in the earliest spectra clearly require separate HVF and PVF components. The phases from -12 days to -8 days reveal contributions from both HVF and PVF. Narrow $\mathrm{Na}$ I $\mathrm{D}$ features are visible in the Si II $\lambda 6355$ profiles. HVF Si III $\lambda 4560$ is blended with PVF Mg II $\lambda 4481$ beginning about -10 days. The velocities measured for each feature are indicated by a blue hashmark for HVF and a red hashmark for PVF. The dotted line at $21,500 \mathrm{~km} \mathrm{~s}^{-1}$ and the dashed line at $13,000 \mathrm{~km} \mathrm{~s}^{-1}$ are the same in all figures.

(A color version of this figure is available in the online journal.)

to be representative of the HVF at -10 days and the PVF near $B$-max. A detailed discussion of individual features is found in Section 4 (HVF) and Section 5 (PVF).

The HVF and PVF of Si II $\lambda 6355$ are easily identified and relatively free from blending. These characteristics make them useful templates that guide efforts to identify absorption features associated with other lines. We compare the velocities, the phases of initial and final detections for HVF and PVF, the relative strengths of the HVF and PVF components at a given phase, and the velocity separation between the HVF and PVF. The presence of simultaneous HVF and PVF from several lines 


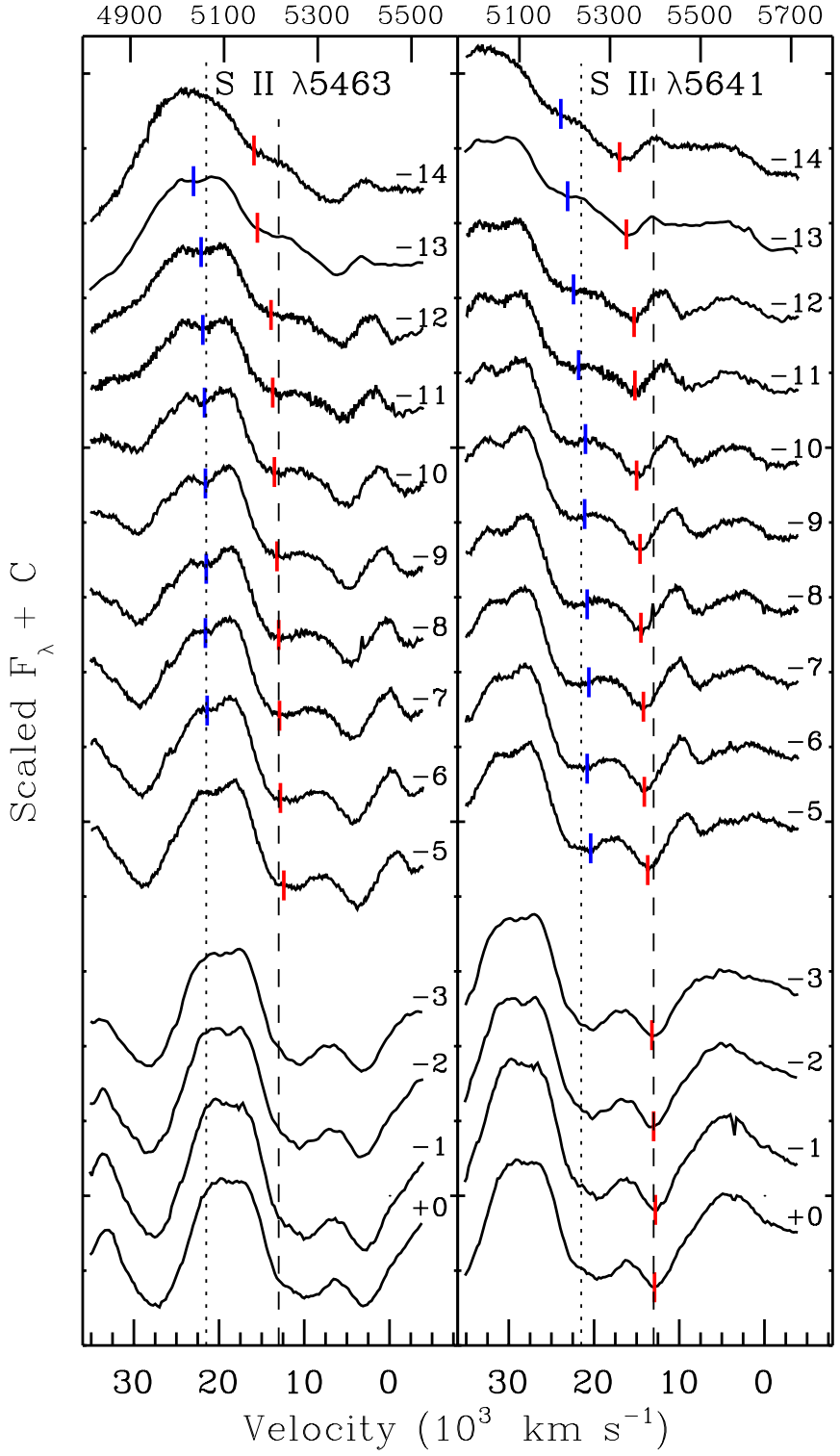

Figure 4. HVF and PVF for S II $\lambda 5453$ (left) and S II $\lambda 5641$ (right) are marked on a sequence of spectra of SN 2009ig obtained between -14 days and +0 days. Rest wavelengths are indicated on the top abscissa. The phase of each spectrum is marked near the red end. HVF are strongest before -10 days and PVF are stronger after -8 days. The measured velocities are indicated by a blue mark for HVF and a red mark for PVF. The PVF in S II $\lambda 5453$ are compromised by blending after -5 days and we do not measure the velocities (see Section 5 ). The dotted line at $21,500 \mathrm{~km} \mathrm{~s}^{-1}$ and the dashed line at $13,000 \mathrm{~km} \mathrm{~s}^{-1}$ are the same in all figures.

(A color version of this figure is available in the online journal.)

increases the number of blended features in the early-time spectra of SN 2009ig. Velocities and line profiles of individual features are measured directly from the flux-calibrated spectra rather than a flattened continuum because the shapes and slopes of the local continua vary widely in different regions of each spectrum.

In the velocity figures, the flux is scaled to make it easier to see details of the absorption features. A representative flux ratio is maintained between the two panels in each figure in order to compare the relative strengths of features from similar lines, but it is not possible to maintain a consistent scale between the figures. The velocity range for the Ca II and Fe II plots 48,000 to $-8000 \mathrm{~km} \mathrm{~s}^{-1}$. For $\mathrm{Si}$ II and $\mathrm{S}$ II velocity plots the range is

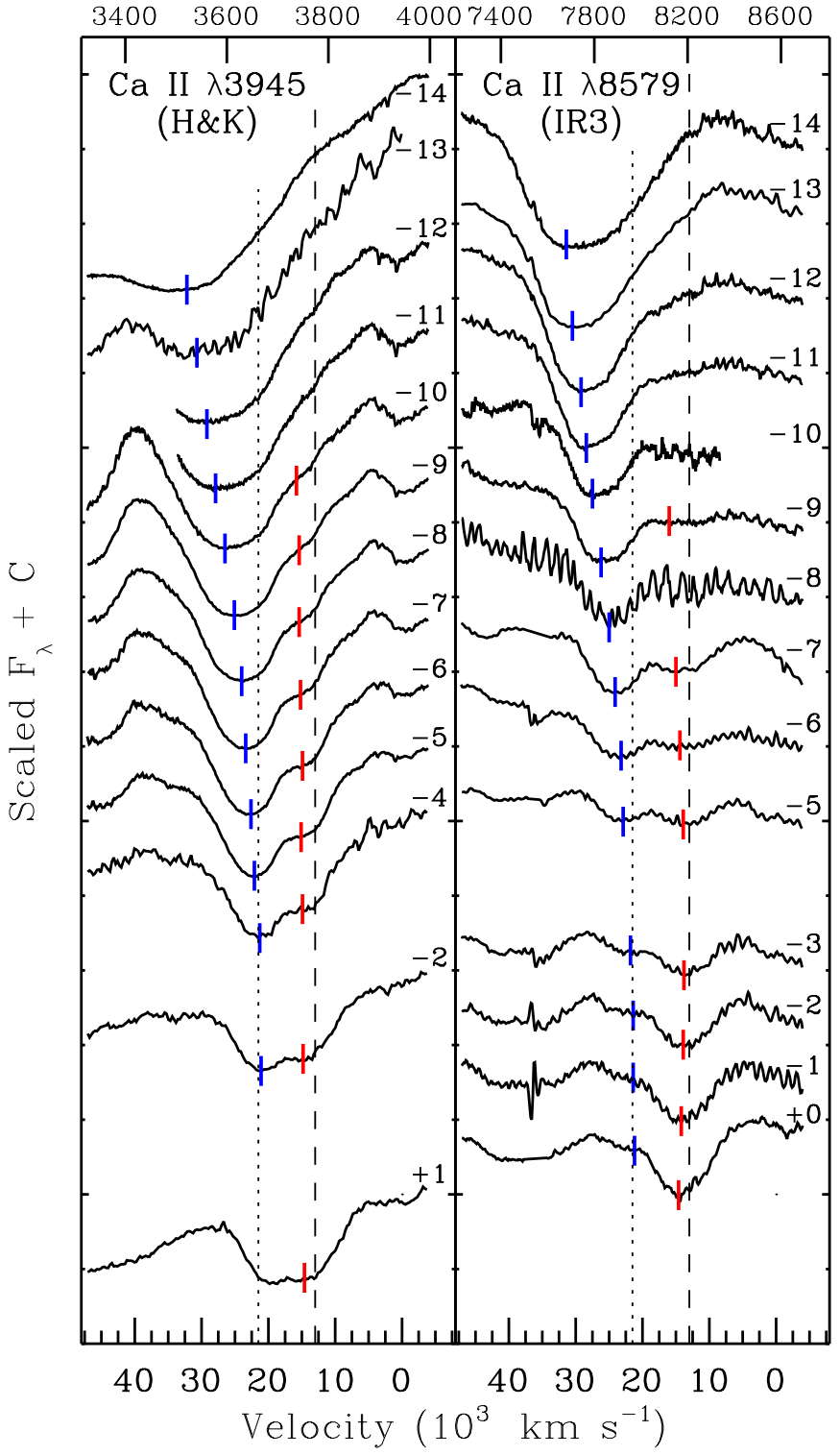

Figure 5. HVF and PVF for Ca II H\&K $\lambda 3945$ (left) and Ca II IR3 $\lambda 8579$ (right) in SN 2009ig are displayed in velocity space (blueshifted velocities are expressed with positive values). Rest wavelengths are indicated on the top abscissa. The spectra were obtained between -14 days and +1 days and the phase of each spectrum is marked near the red end. HVF velocities for Ca II are higher than for other lines in the early spectra (compare offsets from the dotted line), but they eventually decline to the mean HVF velocity for all lines (dotted line). Ca II HVF are detected for a few days longer than HVF for other lines, and the PVF appear later. Comparing the features by phase suggests that the HVF component of Ca II H\&K $\lambda 3945$ is exaggerated by a strengthening contribution from PVF Si II $\lambda 3858$ beginning about -10 days. The measured velocities are indicated by a blue mark for HVF and a red mark for PVF. The dotted line at $21,500 \mathrm{~km} \mathrm{~s}^{-1}$ and the dashed line at $13,000 \mathrm{~km} \mathrm{~s}^{-1}$ are the same in all figures.

(A color version of this figure is available in the online journal.)

36,000 to $-8000 \mathrm{~km} \mathrm{~s}^{-1}$. Throughout this paper we reference blueshifted velocities using positive values.

At many phases one of the components may lack a distinct minimum but still makes an obvious contribution to the overall line profile. For example, the right panel of Figure 3 shows that the PVF component from Si II $\lambda 6355$ at -12 days produces only a small indentation on the red side of the larger absorption feature dominated by HV. As time passes the strength of the HVF signal diminishes and the PVF component becomes stronger. At -9 days the PVF absorption is dominant and the HVF 


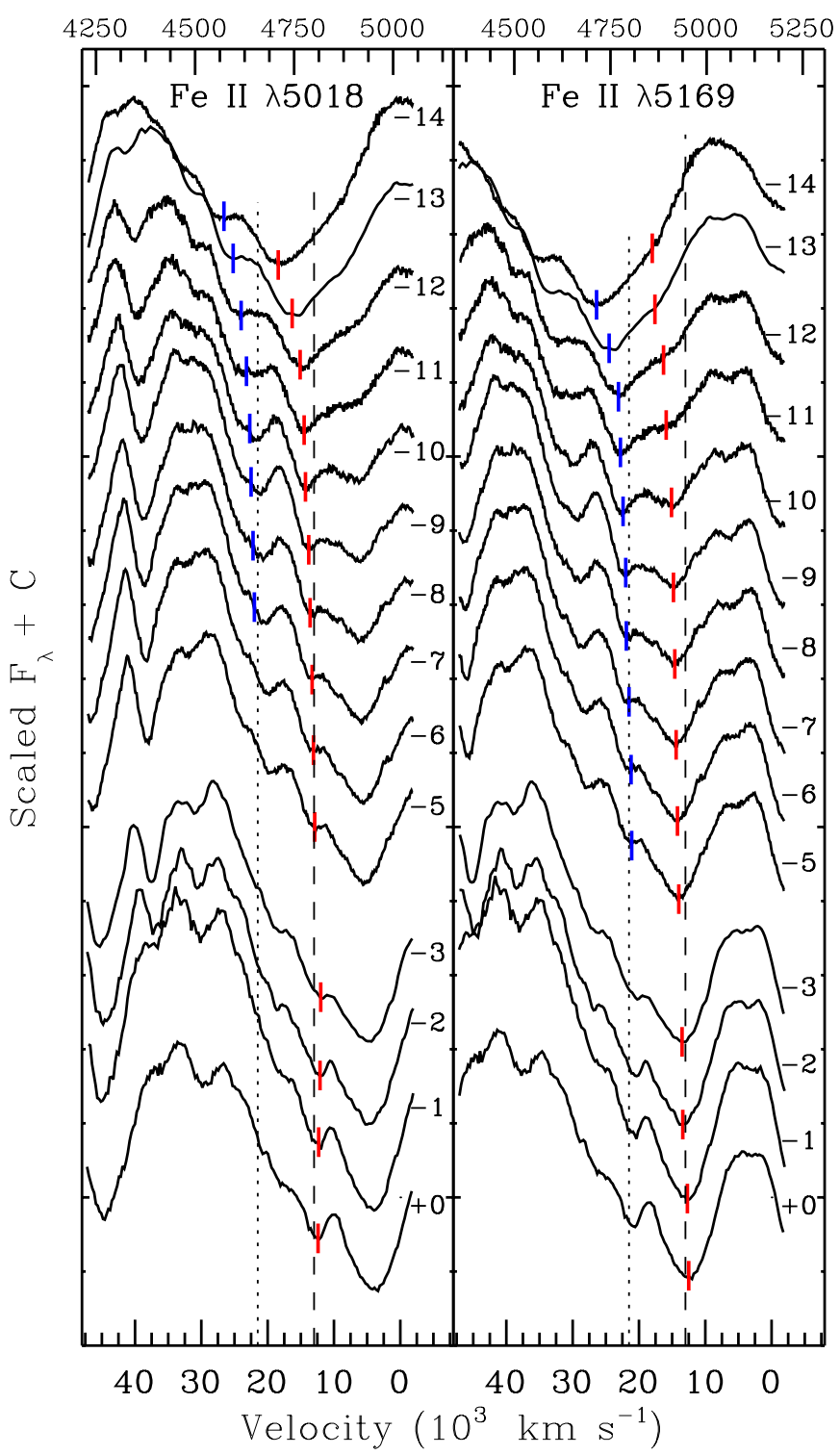

Figure 6. HVF and PVF for Fe II $\lambda 5018$ (left) and Fe II $\lambda 5169$ (right) are marked on a sequence of spectra of SN 2009ig obtained between -14 days and +0 days. Rest wavelengths are indicated on the top abscissa. The phase of each spectrum is marked at the red end. The dashed line in the velocity space of Fe II $\lambda 5018$ and the dotted line in velocity space for HVF Fe II $\lambda 5169$ are in the same physical location. The feature at that position is produced by HVF only in the first two spectra, by a blend of PVF and HVF from about 12 days to -6 days, and by PVF only after -6 days. Comparing offsets from the dotted lines shows that HVF of Fe II are deeper, wider, and at higher velocities than HVF of Si II (Figure 3) and S II (Figure 4). The measured velocities are indicated by a blue mark for HVF and a red mark for PVF. The dotted line at $21,500 \mathrm{~km} \mathrm{~s}^{-1}$ and the dashed line at $13,000 \mathrm{~km} \mathrm{~s}^{-1}$ are the same in all figures.

(A color version of this figure is available in the online journal.)

component forms a small but distinct indentation on the blue side of the PVF absorption feature. We use this spectrum to illustrate a method for measuring velocities from absorption components that form incomplete features.

Figure 7 shows the Si II $\lambda 6355$ feature from the August 27 spectrum ( -9 days). The photospheric component is simply fit by a Gaussian (solid green line) having a minimum at $14,400 \mathrm{~km} \mathrm{~s}^{-1}$. The data in the region of the indentation created by the HVF component are fit with a Gaussian profile having a minimum at $20,700 \mathrm{~km} \mathrm{~s}^{-1}$ (solid blue line). The dotted red lines show alternative Gaussians with minima that have been shifted

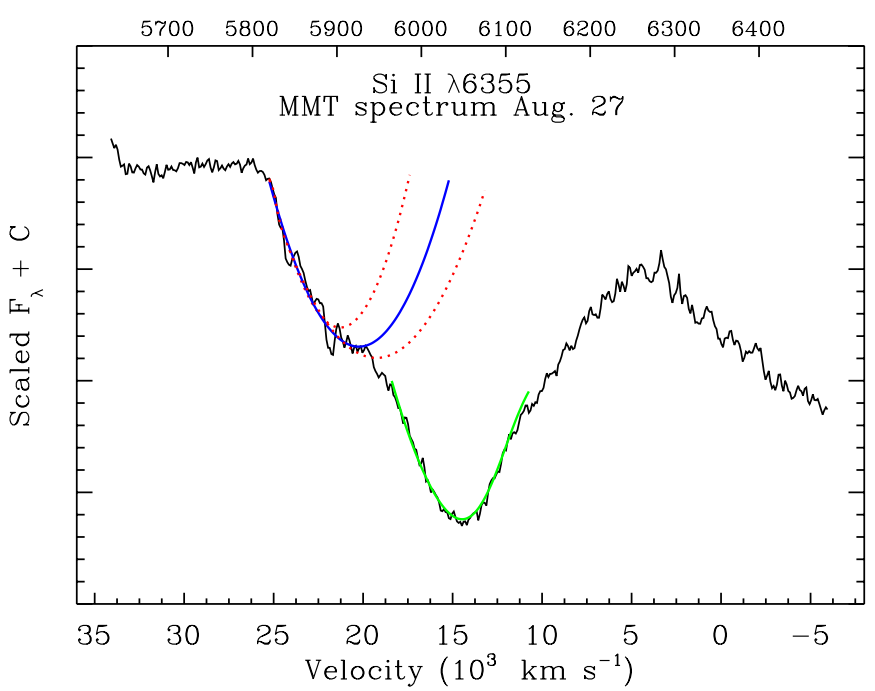

Figure 7. Gaussian profiles are used to measure blended absorption features if one of the components does not produce a distinct minimum. This example shows the Si II $\lambda 6355$ feature in the MMT spectrum of SN 2009ig obtained on August 27 ( -9 days). Rest wavelengths are indicated on the top abscissa. The PVF component is dominant at this phase, but there is clear evidence for a contribution from the HVF component. The solid blue line shows that a Gaussian profile with a minimum at $20,700 \mathrm{~km} \mathrm{~s}^{-1}$ is a good fit to the data. The red dotted lines form Gaussians with minima that have been shifted to 19,700 and $21,700 \mathrm{~km} \mathrm{~s}^{-1}$. The red lines do not fit the data as effectively as the blue line. The photospheric component is fit with a Gaussian (solid green line) having a minimum at $14,400 \mathrm{~km} \mathrm{~s}^{-1}$.

(A color version of this figure is available in the online journal.)

by $\pm 1000 \mathrm{~km} \mathrm{~s}^{-1}$ from the minimum of the blue line. Neither of the line profiles plotted in red fit the data as well as the blue line. The optimal position for the Gaussian is determined by changing the minimum in steps of $100 \mathrm{~km} \mathrm{~s}^{-1}$ and inspecting the results by eye. It is nearly always easy to determine when the Gaussian profile deviates from the actual data by more than a few hundred $\mathrm{km} \mathrm{s}^{-1}$. Uncertainties due to this measurement technique are 100-1000 $\mathrm{km} \mathrm{s}^{-1}$ depending on the quality of the data and how well the minima are defined. The measurements in Tables 2 (HVF) and 3 (PVF) were obtained by this method. Figure 3 illustrates that for Si II $\lambda 6355$, uncertainties will be higher for HVF measured after -9 days and for PVF measured before -11 days.

The SYNOW code (Branch et al. 2003) is another tool for line identification. SYNOW creates model spectra that can compare relative line strengths and positions by wavelength. SYNOW makes it possible to explore the contributions of individual ions to a complete spectrum, and it is particularly helpful for distinguishing between the relative contributions of different lines in a blended feature.

\section{HIGH-VELOCITY ABSORPTION FEATURES $\left(>20,000 \mathrm{~km} \mathrm{~s}^{-1}\right)$}

In most $\mathrm{SNe}$ Ia, Ca II produces the only detectable HVF. In SN 2009ig, eight lines from five ions produce distinct HVF in the early spectra: Si III $\lambda 4560$ and Si II $\lambda 6355$ (Figure 3), S II $\lambda \lambda 5453,5641$ (Figure 4), and Fe II $\lambda \lambda 5018,5169$ (Figure 6). There are also strong suggestions of HVF from several other lines that are associated with blended absorption features for which we are unable to confirm the contributions of individual lines.

HVF are clearly identified from all lines in the -14 days spectrum of SN 2009ig, and HVF remain strong through 
Table 2

High-velocity Features in SN 2009ig $\left(10^{3} \mathrm{~km} \mathrm{~s}^{-1}\right)^{\mathrm{a}}$

\begin{tabular}{lccccccccc}
\hline \hline Date & Phase $^{\mathrm{b}}$ & Ca II & Si III & Fe II & Fe II & S II & S II & Si II & Ca II \\
& & $\lambda 3945$ & $\lambda 4560$ & $\lambda 5018$ & $\lambda 5169$ & $\lambda 5453$ & $\lambda 5641$ & $\lambda 6355$ & $\lambda 8579$ \\
\hline Aug 22 & -14 & 32.2 & 25.7 & 26.6 & 26.4 & $\ldots$ & 23.9 & 22.8 & 31.4 \\
Aug 23 & -13 & 30.7 & $\ldots$ & 25.2 & 24.5 & 23.0 & 23.1 & 22.0 & 30.5 \\
Aug 24 & -12 & 29.2 & 22.8 & 24.0 & 23.1 & 22.1 & 22.4 & 21.1 & 29.2 \\
Aug 25 & -11 & 27.9 & 22.2 & 23.2 & 22.8 & 21.9 & 21.8 & 21.0 & 28.4 \\
Aug 26 & -10 & 26.5 & 22.1 & 22.7 & 22.4 & 21.7 & 21.0 & 20.8 & 27.5 \\
Aug 27 & -9 & 25.1 & 21.7 & 22.5 & 22.0 & 21.6 & 21.1 & 20.7 & 26.2 \\
Aug 28 & -8 & 24.0 & 21.7 & 22.2 & 21.9 & 21.5 & 20.8 & 20.8 & 25.0 \\
Aug 29 & -7 & 23.4 & 21.5 & 22.0 & 21.5 & 21.6 & 20.6 & 20.6 & 24.1 \\
Aug 30 & -6 & 22.6 & 21.3 & $\ldots$ & 21.2 & 21.4 & 20.8 & 20.8 & 23.2 \\
Aug 31 & -5 & 22.1 & 21.1 & $\ldots$ & 21.1 & $\ldots$ & 20.4 & $\ldots$ & 22.9 \\
Sep 1 & -4 & 21.3 & 21.0 & $\ldots$ & $\ldots$ & $\ldots$ & $\ldots$ & $\ldots$ & $\ldots$ \\
Sep 2 & -3 & $\ldots$ & $\ldots$ & $\ldots$ & $\ldots$ & $\ldots$ & $\ldots$ & $\ldots$ & 21.8 \\
Sep 3 & -2 & 21.1 & $\ldots$ & $\ldots$ & $\ldots$ & $\ldots$ & $\ldots$ & $\ldots$ & 21.4 \\
Sep 4 & -1 & $\ldots$ & $\ldots$ & $\ldots$ & $\ldots$ & $\ldots$ & $\ldots$ & $\ldots$ & 21.4 \\
Sep 5 & 0 & $\ldots$ & $\ldots$ & $\ldots$ & $\ldots$ & $\ldots$ & $\ldots$ & $\ldots$ & 21.2 \\
\hline
\end{tabular}

Notes. Measurement uncertainties are higher for HVF after -7 days (see discussion in text).

a Throughout this paper we represent velocities for blueshifted absorption features with positive values.

${ }^{\mathrm{b}}$ Rise time $\tau_{r}=17.1$ days (Foley et al. 2012), so the first spectrum was obtained 2.6 days after the explosion.

-12 days. The strength of the HVF absorptions weaken with time, and in most lines HVF are no longer detectable after -6 days. Beginning at about -10 days, many of the HVF are affected by blends with PVF that are increasing in strength.

The highest velocities for features from each line are measured in the earliest spectrum at -14 days. For Ca II, $v_{\max } \approx$ $32,600 \mathrm{~km} \mathrm{~s}^{-1}$, followed by Fe II at $26,600 \mathrm{~km} \mathrm{~s}^{-1}$, Si III at $25,700 \mathrm{~km} \mathrm{~s}^{-1}, \mathrm{~S}$ II at $23,900 \mathrm{~km} \mathrm{~s}^{-1}$, and Si II at 22,800 $\mathrm{km} \mathrm{s}^{-1}$. This range of velocities for the HVF is apparently caused by differences in the effective opacities of the lines at that phase. By -12 days, the HVF velocities for all lines except Ca II are grouped closely together with a mean of $22,600 \mathrm{~km} \mathrm{~s}^{-1}$. HVF velocities for all lines converge near $21,500 \mathrm{~km} \mathrm{~s}^{-1}$ by -6 days.

\subsection{HVF from Si II, Si III, and $S_{I I}$}

The HVF of SiII $\lambda 6355$ provides a useful guide for the behavior of HVF in general because it is essentially unblended. The HVF component dominates the Si II profile from -14 days to -12 days at velocities that are about $1000 \mathrm{~km} \mathrm{~s}^{-1}$ lower than S II and about $2000 \mathrm{~km} \mathrm{~s}^{-1}$ lower than Fe II. Both HVF and PVF contribute to the Si II $\lambda 6355$ line profile between -12 days and -7 days, and HVF are no longer detected from Si II after -6 days. Narrow $\mathrm{Na}$ I $\mathrm{D}$ features are visible in the spectra at about $23,500 \mathrm{~km} \mathrm{~s}^{-1}$, which is zero velocity for $\mathrm{Na} \mathrm{D}$, and at $24,200 \mathrm{~km} \mathrm{~s}^{-1}$, which is the velocity of the host galaxy, NGC 1015.

Si II has many other lines that often produce detectable features in spectra of SNe Ia. Possible evidence exists for HVF components from Si II $\lambda \lambda 3858,4130,5051$, and 5972, but the features are compromised by blending.

The data do not include sufficiently short wavelengths to cover the HVF of Si III $\lambda 4560$ at -13 days, but this feature is strong at -14 days and -12 days. Contributions from PVF Mg II $\lambda 4481$ and PVF Fe III $\lambda 4407$ begin to affect HVF Si III $\lambda 4560$ by -11 days. We measure HVF Si III until -4 days, when the absorption minimum shifts abruptly to the red, indicating that the HVF contribution has ended. SYNOW models suggest
Table 3

Photospheric Features in SN 2009ig $\left(10^{3} \mathrm{~km} \mathrm{~s}^{-1}\right)^{\mathrm{a}}$

\begin{tabular}{lccccccccc}
\hline \hline Date & Phase $^{\text {b }}$ & Ca II & Si III & Fe II & Fe II & S II & S II & Si II & Ca II \\
& & $\lambda 3945$ & $\lambda 4560$ & $\lambda 5018$ & $\lambda 5169$ & $\lambda 5453$ & $\lambda 5641$ & $\lambda 6355$ & $\lambda 8579$ \\
\hline Aug 22 & -14 & $\ldots$ & 16.7 & 18.4 & 18.0 & 15.9 & 17.0 & $\ldots$ & $\ldots$ \\
Aug 23 & -13 & $\ldots$ & 15.2 & 16.3 & 17.6 & 15.5 & 16.2 & 16.5 & $\ldots$ \\
Aug 24 & -12 & $\ldots$ & 14.0 & 15.1 & 16.3 & 13.9 & 15.3 & 15.9 & $\ldots$ \\
Aug 25 & -11 & $\ldots$ & 13.6 & 14.5 & 15.9 & 13.7 & 15.2 & 15.6 & $\ldots$ \\
Aug 26 & -10 & 15.8 & 13.1 & 14.3 & 15.1 & 13.5 & 15.0 & 15.2 & $\ldots$ \\
Aug 27 & -9 & 15.4 & 12.9 & 13.8 & 14.8 & 13.2 & 14.6 & 14.5 & 16.0 \\
Aug 28 & -8 & 15.4 & 12.8 & 13.6 & 14.6 & 13.0 & 14.5 & 14.2 & $\ldots$ \\
Aug 29 & -7 & 15.2 & 12.4 & 13.3 & 14.4 & 12.9 & 14.2 & 14.1 & 15.0 \\
Aug 30 & -6 & 14.9 & 12.3 & 13.1 & 14.2 & 12.8 & 14.1 & 13.9 & 14.4 \\
Aug 31 & -5 & 15.1 & 11.9 & 12.9 & 14.0 & 12.4 & 13.7 & 13.6 & 13.9 \\
Sep 1 & -4 & 14.9 & 12.1 & $\ldots$ & $\ldots$ & $\ldots$ & $\ldots$ & $\ldots$ & $\ldots$ \\
Sep 2 & -3 & $\ldots$ & $\ldots$ & 12.0 & 13.5 & $\ldots$ & 13.2 & 13.4 & 13.8 \\
Sep 3 & -2 & 14.8 & $\ldots$ & 12.1 & 13.4 & $\ldots$ & 13.0 & 13.3 & 13.9 \\
Sep 4 & -1 & $\ldots$ & $\ldots$ & 12.3 & 12.7 & $\ldots$ & 12.8 & 13.4 & 14.2 \\
Sep 5 & 0 & $\ldots$ & $\ldots$ & 12.4 & 12.5 & $\ldots$ & 12.9 & 13.4 & 14.6 \\
Sep 6 & 1 & 14.6 & $\ldots$ & $\ldots$ & $\ldots$ & $\ldots$ & $\ldots$ & $\ldots$ & $\ldots$ \\
Sep 7 & 2 & $\ldots$ & $\ldots$ & $\ldots$ & $\ldots$ & $\ldots$ & $\ldots$ & $\ldots$ & $\ldots$ \\
Sep 8 & 3 & $\ldots$ & $\ldots$ & $\ldots$ & $\ldots$ & $\ldots$ & $\ldots$ & $\ldots$ & $\ldots$ \\
Sep 9 & 4 & $\ldots$ & $\ldots$ & 12.4 & 11.9 & $\ldots$ & 12.8 & 13.3 & 14.2 \\
Sep 10 & 5 & $\ldots$ & $\ldots$ & 12.6 & 11.8 & $\ldots$ & 13.4 & 13.3 & 14.0 \\
Sep 11 & 6 & 14.3 & $\ldots$ & 12.7 & 11.9 & $\ldots$ & 13.7 & 13.2 & $\ldots$ \\
Sep 12 & 7 & 14.2 & $\ldots$ & 12.8 & 12.0 & $\ldots$ & 13.7 & 13.1 & $\ldots$ \\
Sep 13 & 8 & $\ldots$ & $\ldots$ & $\ldots$ & $\ldots$ & $\ldots$ & 13.2 & $\ldots$ & $\ldots$ \\
Sep 14 & 9 & $\ldots$ & $\ldots$ & 12.7 & 11.9 & $\ldots$ & 13.2 & 13.2 & 14.0 \\
Sep 15 & 10 & 13.9 & $\ldots$ & 12.8 & 12.0 & $\ldots$ & 13.5 & 13.0 & $\ldots$ \\
Sep 16 & 11 & 13.8 & $\ldots$ & 12.8 & 11.9 & $\ldots$ & 13.0 & 13.0 & $\ldots$ \\
Sep 17 & 12 & 13.5 & $\ldots$ & 12.6 & 11.8 & $\ldots$ & $\ldots$ & 12.9 & $\ldots$ \\
Sep 18 & 13 & 13.5 & $\ldots$ & 12.8 & 11.7 & $\ldots$ & $\ldots$ & 13.0 & $\ldots$ \\
\hline & & & & & & & & & \\
\hline
\end{tabular}

Notes. Measurement uncertainties are higher for PVF before -12 days (see discussion in text).

a Throughout this paper we represent velocities for blueshifted absorption features with positive values.

${ }^{\mathrm{b}}$ Rise time $\tau_{r}=17.1$ days (Foley et al. 2012), so the first spectrum was obtained 2.6 days after the explosion.

that the PVF Fe III component is stronger than PVF Mg II from -10 days until about -3 days.

Absorption features in the spectra can possibly be associated with several other doubly ionized lines. Possible HVF are found for Si III $\lambda 5740$, S III $\lambda 4264$, and Fe III $\lambda \lambda 4407,5129$, but as for many other tentative associations, blending prevents definitive identifications.

Figure 4 shows that HVF for both S II $\lambda 5453$ and S II $\lambda 5641$ are stronger at -12 days than they are at -14 days. HVF velocities for S II are between the velocities of HVF Fe II and HVF Si II. The figure also shows that the S II $\lambda 5641 \mathrm{HVF}$ and the S II $\lambda 5453$ PVF form a blended feature. We identify the red component of this blend as HVF S II $\lambda 5641$. The HVF is strongest from -14 days to -12 days, contributions from both HVF and PVF are evident from -10 days to -5 days, and weak absorptions remain near the locations of both $\mathrm{S}_{\mathrm{II}} \mathrm{HVF}$ as late as -3 days. We stop measuring HVF S II $\lambda 5453$ after -6 days and HVF S II $\lambda 5641$ after -5 days. There is possible evidence in the spectra for HVF from $S_{\text {II }} \lambda 5032$ and $\lambda 5429$.

HVF are not detected from carbon, oxygen, or magnesium, so there is no evidence for unburned fuel or carbon burning products in the HVF region of SN 2009ig. If the Mg II $\lambda 4481$ line produced a HVF at -14 days, it would have a velocity of about $24,000 \mathrm{~km} \mathrm{~s}^{-1}$, and it would appear at about $29,000 \mathrm{~km} \mathrm{~s}^{-1}$ in the velocity space of Si III $\lambda 4560$ (left panel of 
Figure 3$)$. At -10 days, the Mg II HVF velocity would be about $21,500 \mathrm{~km} \mathrm{~s}^{-1}$, corresponding to $26,500 \mathrm{~km} \mathrm{~s}^{-1}$ in the figure. The spectra are steeply sloped toward the red in this region, which makes it more difficult to discern small absorptions, but careful inspection reveals no evidence of absorption features that can be associated with HVF from Mg II.

\subsection{HVF from $\mathrm{Ca}$ II and $\mathrm{Fe}$ II}

The velocity plots and tables show that HVF of Ca II and Fe II are wider and deeper, with absorption minima at higher velocities than the HVF of other lines. Foley et al. (2012) observed that HVF of both CaII lines are stronger and have higher velocities than HVF of any other lines in the spectra of SN 2009ig.

The Fe II $\lambda 5169$ HVF produces a very strong absorption from -14 days to -11 days that is nearly as broad and deep as the Ca II HVF. The feature is subsequently affected by PVF Fe II $\lambda 5018$, PVF Si II $\lambda 5051$, and PVF S II $\lambda 5032$ beginning about -10 days. We stop measuring HVF Fe II $\lambda 5169$ after the PVF blend becomes dominant at -5 days.

HVF Fe II $\lambda 5018$ is the strongest part of a blend with the HVF components of S II $\lambda 5032$ and Si II $\lambda 5051$ from -14 days to about -10 days. This identification is confirmed by the close agreement of the measured velocities of HVF Fe II $\lambda 5169$ and HVF Fe II $\lambda 5018$. This feature loses a distinct minimum after -7 days and we stop measuring HVF Fe II $\lambda 5018$ at that point. Possible evidence exists for HVF components from Fe II $\lambda \lambda 4924,5129$.

Figure 5 displays the Ca II H\&K blend $\lambda 3935$ (left) and the Ca II IR3 $\lambda 8579$ (right). At -14 days, the absorption minima for both $\mathrm{Ca}$ II blends are near $32,000 \mathrm{~km} \mathrm{~s}^{-1}$ and the features have blue wings that extend beyond $40,000 \mathrm{~km} \mathrm{~s}^{-1}$. Simultaneous observations of CaII H\&K and IR3 are of particular interest as they provide a rare opportunity to compare the evolution of these features. As shown in Figure 5 and Table 2, the measured velocities and rates of change are very similar for $\mathrm{HVF}$ from both of the strong Ca II blends. Although Ca II HVF velocities are about $7000 \mathrm{~km} \mathrm{~s}^{-1}$ higher than Fe II HVF and nearly $10,000 \mathrm{~km} \mathrm{~s}^{-1}$ higher than Si II HVF at -14 days, they have a significantly faster decline rate, and the HVF velocities for all lines converge near $21,500 \mathrm{~km} \mathrm{~s}^{-1}$ at -6 days.

HVF from the Ca II IR3 are unblended, and they are weak but detectable until $B$-max. That is about 5 days after the last HVF detection from other lines. The HVF component of Ca II H\&K $\lambda 3945$ is blended with PVF Si II $\lambda 3858$. Side by side comparison of the CaII H\&K and IR3 line profiles suggests that the HVF from Ca II H\&K are broadened by Si II PVF beginning about -10 days and the PVF influence gets stronger with time. We find that -2 days is the final phase at which the absorption minimum of the $\mathrm{Ca} / \mathrm{Si}$ blend is consistent with the HVF of Ca II IR3, so we stop measuring Ca II H\&K HVF after that phase.

Foley et al. (2013) demonstrated that there are conditions under which the absorption feature attributed to HVF Ca II H\&K can be produced by a combination of Si II and a particular density profile for Ca II. Wang et al. (2006) used polarization data in a -6 days spectrum from SN 2004dt to suggest that Si II provides the dominant contribution to this blend at -6 days.

Childress et al. (2013a) compare the average strength of the Ca II HVF component in spectra from SNe Ia at $B$-max. They find that slowly declining SNe Ia produce either high PVFs or strong HVF Ca II close to $B$-max, but not both. SN 2009ig fits this pattern with $v_{\mathrm{Si}}=13,400 \mathrm{~km} \mathrm{~s}^{-1}$, while the Ca II IR3 series in Figure 5 shows that HVF Ca II is very weak at $B$-max.

\section{PHOTOSPHERIC-VELOCITY ABSORPTION FEATURES}

We use velocity offsets from PVF and their relative strengths by phase to confirm the identifications of HVF in spectra of SN 2009ig. PVF are identified for each of the same lines that produce HVF detections: Si III $\lambda 4560$ and Si II $\lambda 6355$ (Figure 3), S II $\lambda \lambda 5453,5641$ (Figure 4), and Fe II $\lambda \lambda 5018,5169$ (Figure 6). PVF velocity measurements are in Table 3.

There are weak suggestions of PVF for some lines in the -14 days spectrum of SN 2009ig, but the first unambiguous detections of PVF are at -12 days. Foley et al. (2012) note that -12 days is the phase at which the rate of change slows down for $B-V$ colors. PVF become progressively stronger with time, and the PVF of most lines are stronger than the HVF by about -10 days. At -6 days the PVF components dominate all blended absorption features with little or no influence from the HVF.

PVF velocities in SN 2009ig decline steeply at first and then more gradually. They become essentially constant after -4 days. This behavior is consistent with the typical pattern for PVF velocities in SNe Ia (Foley et al. 2011; Silverman et al. 2012). The PVF velocities of SN 2009 ig are about $2000 \mathrm{~km} \mathrm{~s}^{-1}$ greater than velocities in a "normal" SN Ia, but they are not exceptional. We measure $v_{\mathrm{Si}}=13,400 \mathrm{~km} \mathrm{~s}^{-1}$, which places SN 2009ig in the $H V$ group defined by Wang et al. (2009a). Foley et al. (2012) show that $v_{\mathrm{Si}}<13,500 \mathrm{~km} \mathrm{~s}^{-1}$ for $\sim 85 \%$ of SNe Ia having $\Delta m_{15}(B) \leqslant 1.5 \mathrm{mag}$. The change in Si II $\lambda 6355$ velocity between +0 days and +10 days is $\sim 40 \mathrm{~km} \mathrm{~s}^{-1}$ per day. That puts SN 2009ig in the Low Velocity Gradient group defined by Benetti et al. (2005).

\subsection{Individual PVF}

PVF Si II $\lambda 3858$ is blended with HVF Ca II H\&K. The Si II contribution is significant as early as -10 days, but it is not clear when the Si II PVF become strong enough to define the minimum in this feature. We do not take velocity measurements from this line. PVF Si II $\lambda 4130$ is the probable source of the absorption visible as a small notch near $4000 \AA$ in the P Cygni emission peak from Ca II H\&K. HVF S III $\lambda 4264$ is a likely contributor to this feature. PVF of Si II $\lambda \lambda 5051,5972$ are possibly identified.

Most of the Fe II PVF are strongly affected by blending with other PVF. PVF Fe II $\lambda 5018$ (left panel Figure 6) is blended with PVF Si II $\lambda 5051$ and PVF S II $\lambda 5032$. The Si II and S II lines may pull the observed minimum of this feature to the red since the PVF velocities for Fe II $\lambda 5018$ before $B$-max are $1000-1500 \mathrm{~km} \mathrm{~s}^{-1}$ lower than PVF Fe II $\lambda 5169$.

The strong absorption feature near $4950 \AA$ is formed by PVF $\mathrm{Fe}$ II $\lambda 5169$ with contributions from PVF Fe II $\lambda 5266$ and PVF Fe III $\lambda 5129$. The blend of PVF Fe II $\lambda 5018$, Si II $\lambda 5051$, and S II $\lambda 5032$ is observed near $4800 \AA$, and this feature continues to grow stronger after $B$-max. The P Cygni emission component of this feature pushes the minimum for PVF Fe II $\lambda 5169$ to velocities that are about $1000 \mathrm{~km} \mathrm{~s}^{-1}$ lower than PVF Fe II $\lambda 5018$ and other PVF lines after $B$-max.

PVF S II $\lambda 5453$ is blended with HVF S II $\lambda 5641$ from -12 days to -9 days. We stop measuring PVF S II $\lambda 5453$ after -5 days when the velocity abruptly drops from $12,400 \mathrm{~km} \mathrm{~s}^{-1}$ at -5 days to $10,400 \mathrm{~km} \mathrm{~s}^{-1}$ at -3 days. That is not consistent with the other PVF lines at this phase including the other S II line at $\lambda 5641$. The displacement of the PVF $\lambda 5453$ feature suggests that it is also moved to the red by $\mathrm{P}$ Cygni emission from the strong blend of Fe-group lines observed at $4950 \AA$. 
PVF from lines of doubly ionized ions are weak at -14 days and -13 days, become significantly stronger at -12 days, and disappear at about -3 days. Figure 3 shows this pattern for PVF Si III $\lambda 4560$. Other possible but unconfirmed identifications of PVF from twice-ionized lines show similar behavior: Si III $\lambda 5740, S_{\text {III }} \lambda 4264$, and Fe III $\lambda \lambda 4407,5129$. The HVF components of S II lines also appear to be stronger at -12 days than at -14 days.

PVF Mg II $\lambda 4481$ is a major contributor to the very strong absorption found near $4220 \AA$ in a blend with PVF Fe III $\lambda 4407$ and HVF Si III $\lambda 4560$. The left panel of Figure 3 shows the minimum of this feature near the dotted line at $21,500 \mathrm{~km} \mathrm{~s}^{-1}$ until -3 days. A Mg II PVF with a velocity of $14,000 \mathrm{~km} \mathrm{~s}^{-1}$ would appear at about $19,000 \mathrm{~km} \mathrm{~s}^{-1}$ on the velocity scale of Si III $\lambda 4560$. SYNOW models show a better fit to the data when the PVF Fe III component is stronger than Mg II from -10 days until about -3 days. The SYNOW result is consistent with the abrupt shift of this feature to the red at -3 days that aligns the minimum with characteristic PVF velocities for $\mathrm{Mg}$ II $\lambda 4481$.

The evidence for PVF from unburned C and O in SN 2009ig is inconclusive. C II $\lambda 4743$ makes a reasonable fit to absorption features for both HVF near $4370 \AA$ and PVF near $4480 \AA$ (Figure 3, wavelength axis on top). If, however, $C_{\text {II }} \lambda 4743$ is the source of these features, then C II $\lambda 6580$ should also be easily detected. The PVF component of C II $\lambda 6580$ would appear near $3000 \mathrm{~km} \mathrm{~s}^{-1}$ in the velocity space of Si II $\lambda 6355$. Foley et al. (2012) cite work with SYNOW models (Parrent et al. 2011) that finds C II $\lambda 6580$ to be present in the early-time spectra of SN 2009ig. C II is purported to cause a flattening of the red wing in the line profile of $\mathrm{Si}$ II $\lambda 6355$ from -14 days to -12 days. It may be that including $\mathrm{C}_{\text {II }}$ improves the SYNOW fit to the line profile, but we do not find any absorption features at this location; the tiny notch at $6200 \AA$ is telluric.

O I $\lambda 7773$ develops a broad PVF line beginning about -3 days, but it is much weaker than PVF OI found in many SNe Ia. The features near $7400 \AA$ in the spectra from -3 days to +9 days correspond to PVF of O I $\lambda 7773$ at $11,000-12,000 \mathrm{~km} \mathrm{~s}^{-1}$, consistent with other PVF velocities those phases.

\section{THE LOCATION OF HVF LINE FORMING REGIONS}

Figure 8 can be used to trace the location of the line forming regions for $\mathrm{HVF}$ of $\mathrm{Ca}$ II $\mathrm{H} \& \mathrm{~K}, \mathrm{Ca}$ II IR3, Fe II $\lambda 5169$, and $\mathrm{Si}$ II $\lambda 6355$ at phases -14 days, -12 days, -10 days, -6 days, and +0 days (top to bottom). Velocity serves as a proxy for radial distance, with the center of the SN on the right at $0 \mathrm{~km} \mathrm{~s}^{-1}$ and the radius increasing with velocity from right to left.

The spectra are normalized to 1.0 at $6500 \AA$ and the features are normalized to a flat continuum. The flux scale is the same in each panel. At -14 days, all features have the same flux calibration. At later phases, all other features retain the same flux calibration, but $\mathrm{Ca}$ II $\mathrm{H} \& \mathrm{~K} \mathrm{HVF}$ is scaled down to fit in the figure.

At -14 days, both Ca II HVF (purple and red) occupy the same velocity space, and a blue wing is present in both features that extends from 40,000 to $\sim 55,000 \mathrm{~km} \mathrm{~s}^{-1}$. The minima for the Ca II HVF are at velocities greater than $30,000 \mathrm{~km} \mathrm{~s}^{-1}$ (Table 2 and Figure 9). The HVF velocity from Fe II $\lambda 5169$ is near $26,000 \mathrm{~km} \mathrm{~s}^{-1}$. The line profile for Fe II at this phase is similar to that of Ca II H\&K in both width and depth. A velocity difference of about $5000 \mathrm{~km} \mathrm{~s}^{-1}$ is evident between the Ca II line forming region (purple line) and Fe II line forming region (blue line) on the red sides of the features. The blue sides, however,

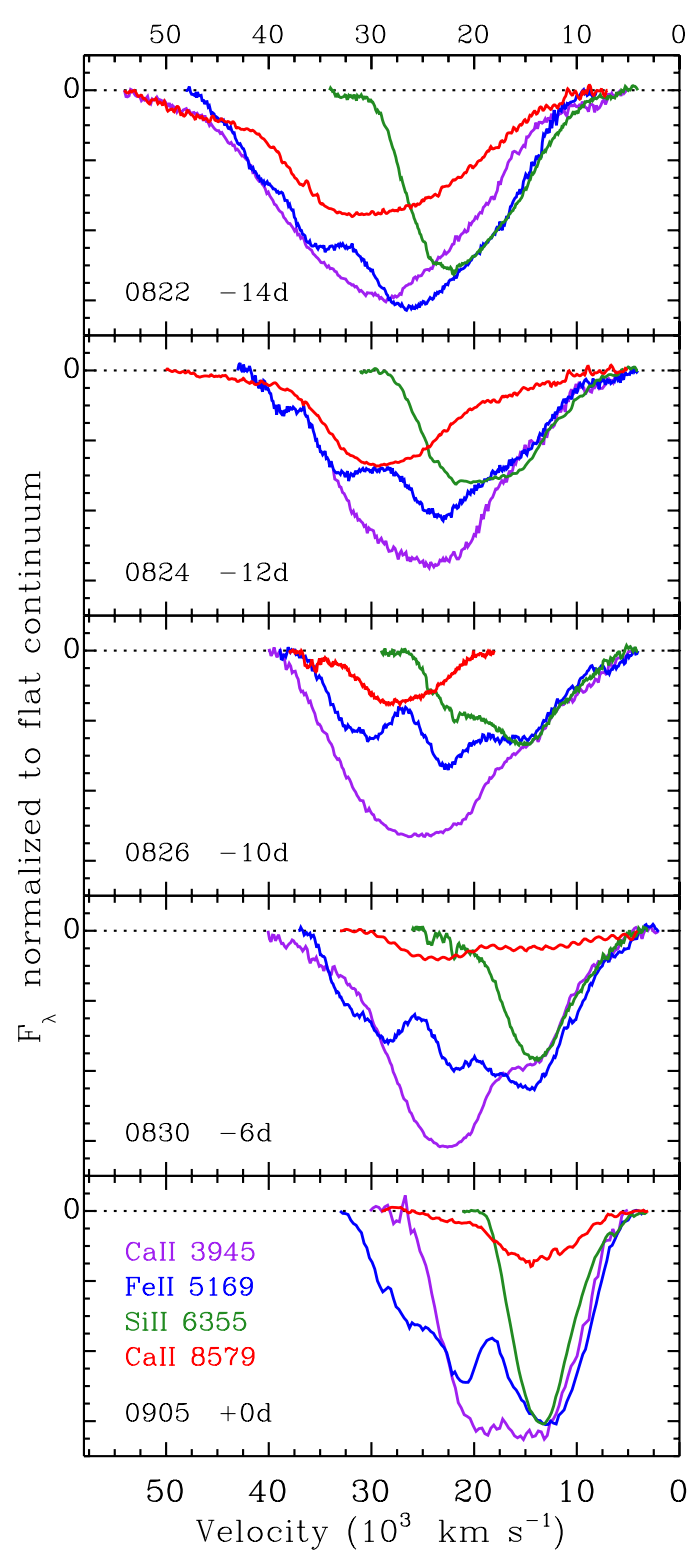

Figure 8. HVF of Ca II H\&K (purple), Ca II IR3 (red), Fe II $\lambda 5169$ (blue), and Si II $\lambda 6355$ (green) are plotted together in velocity space at phases -14 days, -12 days, -10 days, -6 days, and +0 days (top to bottom). Both HVF of Ca II occupy the same physical space at -14 days. The Fe II HVF has a similar profile with an offset of about $7000 \mathrm{~km} \mathrm{~s}^{-1}$. The HVF of Fe II $\lambda 5018$ forms the notch near $35,000 \mathrm{~km} \mathrm{~s}^{-1}$. At -14 days, the Si II HVF is confined to the lower velocity half of the Fe II region. At -12 days, HVF remain strong from all lines and weak PVF are detected from Si II and Fe II. Weakening HVF of Si II and Fe II are still detected at -10 days, and Ca II HVF remain strong. At this phase HVF Fe II $\lambda 5169$ is affected by blending with PVF Fe II $\lambda 5018$, and HVF Ca II H\&K is blended with $\mathrm{Si}$ II $\lambda 3858$. At -6 days, the only evidence for HVF is very weak Ca II, and at +0 days, all absorptions are PVF. The HVF of Si II ( -14 days) are asymmetric with steeper blue sides while PVF of Si II ( -6 days, +0 days) are nearly symmetric.

(A color version of this figure is available in the online journal.)

are closely aligned. This discrepancy is caused by the HVF of Fe II $\lambda 5018$ that creates a notch observed near $35,000 \mathrm{~km} \mathrm{~s}^{-1}$ in the velocity space of Fe II $\lambda 5169$ (blue line). Between the red edge of this notch and the continuum, the side of the Fe II $\lambda 5169$ HVF is shifted by about $7000 \mathrm{~km} \mathrm{~s}^{-1}$ to the blue.

The Si II HVF at -14 days has a minimum at about $23,000 \mathrm{~km} \mathrm{~s}^{-1}$, and the line forming region is confined to a velocity space that is almost entirely in the lower velocity half of the Fe II HVF. The Si II line profile at -14 days (green) is 


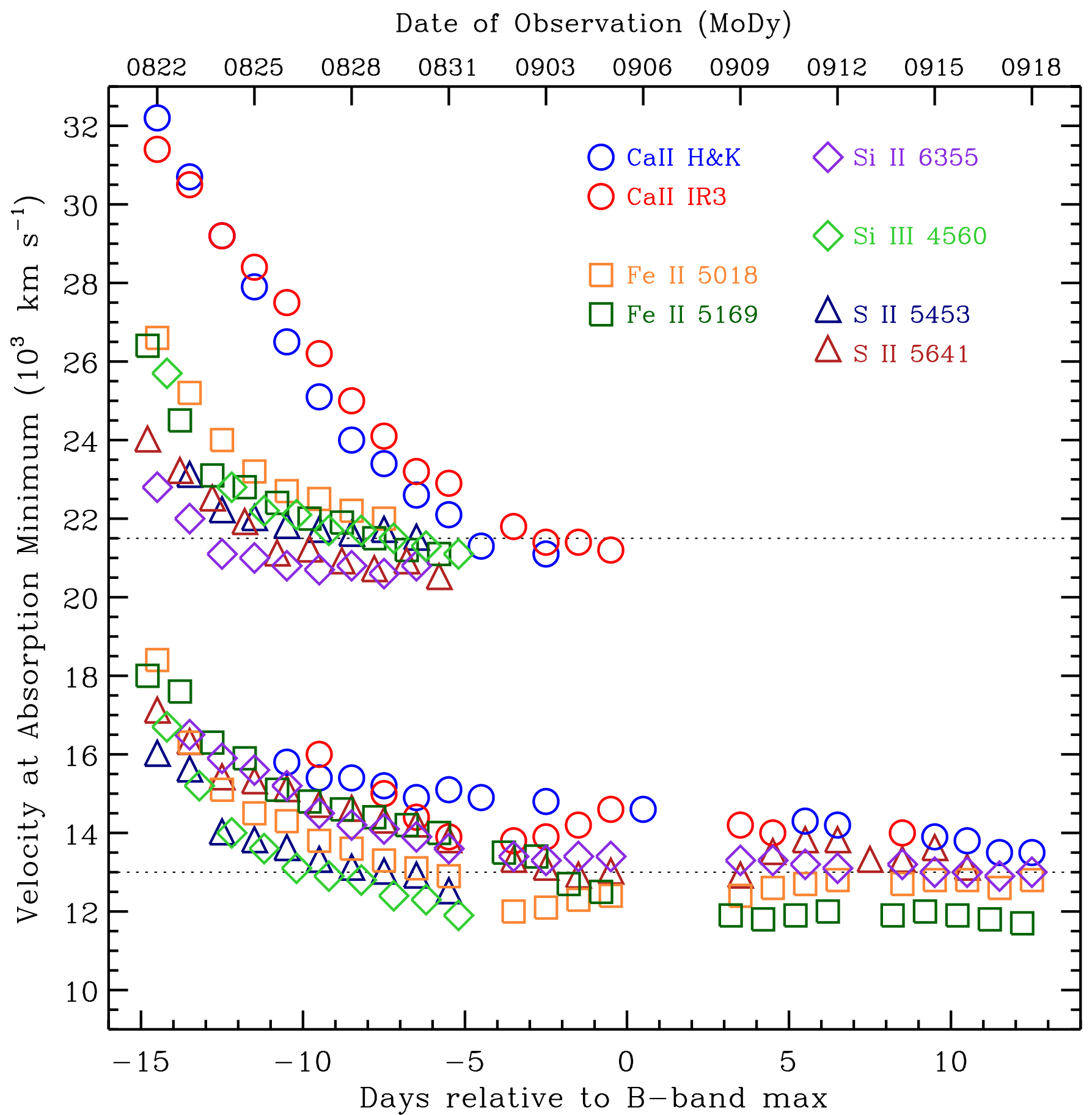

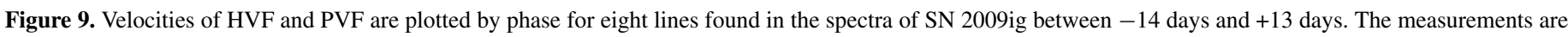

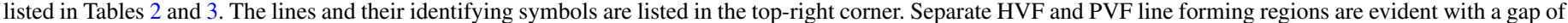

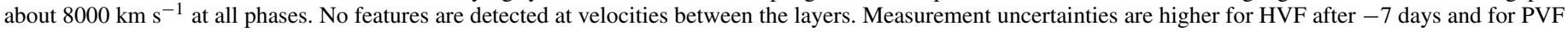

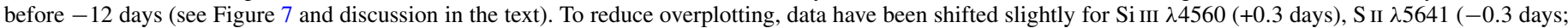
HVF only), and Fe II $\lambda 5169$ ( -0.3 days).

(A color version of this figure is available in the online journal.)

clearly asymmetric, with a steeper blue side and an abrupt transition at the blue edge from the absorption to the continuum. The blue limit of the Si II HVF has nearly the same velocity as the absorption minimum for Ca II. In contrast, the PVF of Si II ( -6 days, +0 days) are nearly symmetric.

At -12 days, HVF are strong from all lines, with velocities for Ca II about 29,000 $\mathrm{km} \mathrm{s}^{-1}$, for Fe II near 23,000 km s $\mathrm{km}^{-1}$, and for Si II near $21,000 \mathrm{~km} \mathrm{~s}^{-1}$. The Si II HVF is again formed in a smaller region than for other lines. The blue limit of the Si II HVF is close to the same velocity as it was at -14 days. PVF from Si II and Fe II can be detected near 16,000 $\mathrm{km} \mathrm{s}^{-1}$ at this phase, with the Si II PVF significantly distorting the line profile.

At -10 days, the HVF of Si II is noticeably weaker, but it is clearly present as it forms the distortion in the blue side of the Si II PVF. Using the methods described in Section 3, the velocity 
of HVF from Si II is measured to be near $21,000 \mathrm{~km} \mathrm{~s}^{-1}$. The HVF velocity of Fe II is near $22,000 \mathrm{~km} \mathrm{~s}^{-1}$ and the Ca II HVF remains strong at about $27,000 \mathrm{~km} \mathrm{~s}^{-1}$. At this phase, the HVF of Fe II $\lambda 5169$ has a significant contribution from PVF Fe II $\lambda 5018$ that narrows the observed profile. The Ca II H\&K HVF also receives a strong contribution from PVF Si II $\lambda 3858$.

At -6 days, the most obvious evidence for HVF is a very weak absorption from the Ca II IR3 HVF. The blue line absorption near $21,000 \mathrm{~km} \mathrm{~s}^{-1}$ is primarily due to the PVF of Fe II $\lambda 5018$. The Ca II H\&K HVF absorption (purple) near 23,000 $\mathrm{km} \mathrm{s}^{-1}$ is a blend with PVF Si II $\lambda 3858$. The magnitude of the contribution from Si II can be estimated by comparing the HVF of Ca II $\mathrm{H} \& \mathrm{~K}$ to the $\mathrm{HVF}$ of $\mathrm{Ca}$ II IR3. The broad $\mathrm{Ca} / \mathrm{Si}$ profile does not provide a HVF detection. At this phase, Si II has a PVF at $14,000 \mathrm{~km} \mathrm{~s}^{-1}$, and the line profile is nearly symmetric in velocity space with no evidence of a HVF.

Figure 8 shows that the absorption minima for HVF are always observed above $20,000 \mathrm{~km} \mathrm{~s}^{-1}$. There is no evidence at any phase for HVF with lower velocities. By +0 days, all absorptions are easily explained by associations with PVF at velocities near $13,000 \mathrm{~km} \mathrm{~s}^{-1}$. The blue wing of the CaII IR3 PVF at this phase is slightly flattened by the remnant of the HVF.

Figure 9 plots HVF and PVF velocity measurements by phase for Si II $\lambda 6355$, Si III $\lambda 4560$, S II $\lambda \lambda 5453,5641$, Ca II $\lambda \lambda 3945$, 8579 , and Fe II $\lambda \lambda 5018,5169$. All lines contribute both HVF and PVF measurements to the figure. The plotted data are found in Tables 2 and 3. This figure is used to visually compare the relative velocities of both $\mathrm{HVF}$ and PVF from different lines at the same phases. Variations in the decline rates are easy to discern. HVF and PVF from the same lines or the same ions can also be compared.

HVF velocities converge to a common value near $21,500 \mathrm{~km} \mathrm{~s}^{-1}$ and PVF velocities are grouped together at all phases. A significant gap in velocity space is evident between the HVF and PVF regions for all lines at all phases. The separation is about $8,000 \mathrm{~km} \mathrm{~s}^{-1}$, except Ca II which displays a greater separation before -5 days and $\mathrm{Si}$ II $\lambda 6355$ for which the separation at all phases is about $7000 \mathrm{~km} \mathrm{~s}^{-1}$. No features are found at velocities that would place them between these layers.

Figure 9 shows that from -14 days to -10 days, the HVF velocities of both $\mathrm{Fe}$ II lines are intermediate between the higher Ca II HVF velocities and the lower HVF velocities for other lines. By -9 days, Fe II HVF velocities are essentially the same as the velocities of other HVF that are not Ca II.

The patterns of velocity by phase can be compared the behavior of the HVF line profiles by phase that are displayed in Figure 8. Si II and Fe II both have their maximum velocities in the earliest spectrum at -14 days. At that phase, the Fe II HVF line profile is wide, deep and more like the HVF of Ca II then the HVF of Si II. The velocity difference between the HVF of Fe II and Si II is $3600 \mathrm{~km} \mathrm{~s}^{-1}$. At -10 days, the HVF line profiles are less distinct due to blending with PVF. The Fe II line profile is narrower and weaker and the velocities of Si II and Fe II are separated by $1600 \mathrm{~km} \mathrm{~s}^{-1}$. The HVF of Ca II at this phase remain broad and at higher velocities. At -6 days, the HVF of $\mathrm{Si}$ II and Fe II are very weak and their measured velocities are within $400 \mathrm{~km} \mathrm{~s}^{-1}$.

The velocity dispersion of the HVF components of Si II, $\mathrm{Si}$ III, $\mathrm{S}$ II, and $\mathrm{Fe}$ II is confined within a narrow velocity region of less than $2000 \mathrm{~km} \mathrm{~s}^{-1}$ from -12 days until HVF are no longer detected. By -9 days, the mean $\mathrm{HVF}$ velocity (excluding $\mathrm{Ca}$ II) is about $21,500 \mathrm{~km} \mathrm{~s}^{-1}$, and Ca II velocities

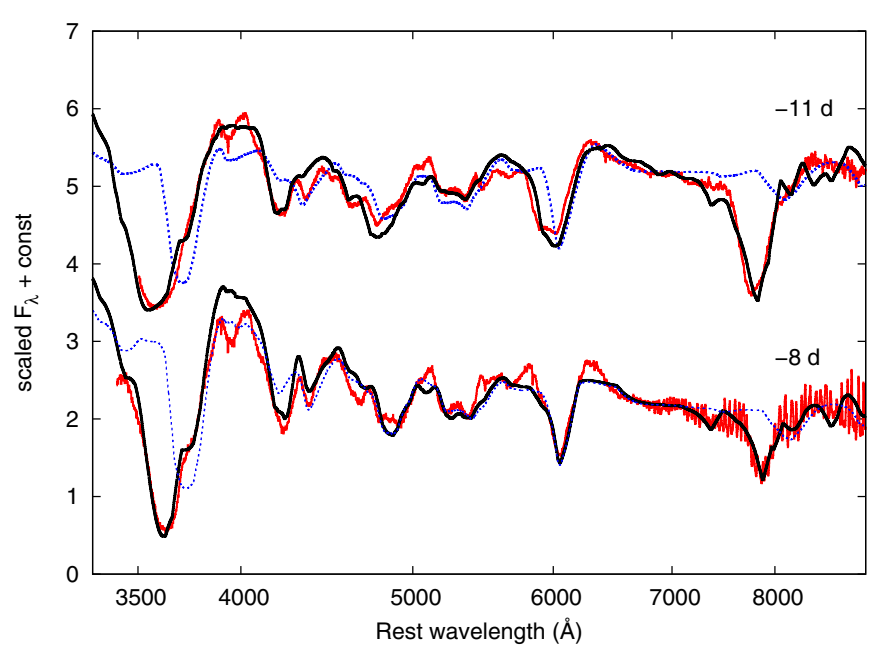

Figure 10. Spectra from SN 2009ig obtained at -11 days and -8 days are plotted with spectra from SYNOW models. The data are shown in red. Model spectra with only PVF are dotted blue and model spectra with both PVF and HVF are solid black. The addition of HVF lines clearly improves the agreement between the model and the data.

(A color version of this figure is available in the online journal.)

reach this value a few days later. After the HVF velocities reach $\sim 21,500 \mathrm{~km} \mathrm{~s}^{-1}$, the velocities remain constant within the range of scatter introduced by measurement uncertainties. The mean PVF velocity is observed to be about $13,000 \mathrm{~km} \mathrm{~s}^{-1}$ at -4 days, and it declines less than $1000 \mathrm{~km} \mathrm{~s}^{-1}$ through the end of our observations at +13 days. (Most of the decline is caused by Fe II $\lambda 5169$; see Section 5.) The relative velocities of the HVF and PVF regions imply that, with homologous expansion, the radius of the HVF line forming region is $\sim 1.6$ times greater than the radius of the PVF line forming region.

Figure 10 shows SYNOW model spectra plotted with data from SN 2009ig obtained at -11 days and -8 days. These phases are chosen because they include HVF and PVF, and the relative strengths of the components change in the time interval. The data are plotted in the figure with red lines. SYNOW spectra from models with only PVF velocities are plotted in blue with dotted lines, and model spectra with both HVF and PVF are solid black. At both phases, the addition of HVF noticeably improves the models in the regions of the $\mathrm{Ca}$ II and $\mathrm{Si}$ II features. More subtle improvements are evident around the Fe II and Si III features.

The SYNOW models using only PVF velocities produce a Ca II IR3 feature that is weak and shifted to longer wavelengths because the $\mathrm{Ca}$ II lines at -11 days and -8 days are dominated by the HVF component. The relative weakness of the PVF Ca II IR3 feature in the model is a consequence of SYNOW setting the optical depth for each ion to represent the strongest line (in this case $\mathrm{Ca}$ II $\mathrm{H} \& \mathrm{~K}$ ) and then calculating the strength of other lines from that ion assuming local thermodynamic equilibrium. The SYNOW parameters for the models are given in Table 4. In these simple models, the same HVF and PVF velocities are assigned to all ions except for HVF Ca II and Fe II.

Figure 10 can be compared to Figure 4 of Branch et al. (2005), which uses HVF Fe II to improve the SYNOW fit to a -12 days spectrum from SN 1994D. Additional HVF contributions from $\mathrm{Mg}$ II and Fe III can be added to further improve the agreement between SYNOW model spectra and the data, but HVF from these lines are not clearly identified in SN 2009ig. Mg II and Fe III are not included in the velocity tables or plots. 
Table 4

SYNOW Parameters for Model Spectra

\begin{tabular}{|c|c|c|c|c|c|c|c|c|}
\hline Ion & \multicolumn{4}{|c|}{$\begin{array}{l}-11 \text { day } \\
=16,000 \mathrm{~km} \mathrm{~s}^{-1}\end{array}$} & \multicolumn{4}{|c|}{$\begin{array}{c}-8 \text { day } \\
v_{\text {phot }}=13,800 \mathrm{~km} \mathrm{~s}^{-1}\end{array}$} \\
\hline Si II & 3.0 & 16 & 30 & 2.0 & 1.2 & 16 & 30 & 2.0 \\
\hline Si II(HVF) & 1.0 & 23 & 50 & 2.0 & 0.1 & 23 & 50 & 2.0 \\
\hline Si III & 0.8 & 16 & 50 & 2.0 & 0.8 & 13 & 50 & 2.0 \\
\hline Si III(HVF) & 0.5 & 23 & 50 & 2.0 & 0.5 & 23 & 50 & 2.0 \\
\hline Mg II(HVF) & 0.5 & 22 & 50 & 4.0 & 0.3 & 22 & 50 & 4.0 \\
\hline $\mathrm{S}_{\text {II }}$ & 0.7 & 16 & 50 & 2.0 & 0.5 & 13 & 50 & 2.0 \\
\hline S II(HVF) & 0.1 & 23 & 50 & 2.0 & 0.1 & 23 & 50 & 2.0 \\
\hline Ca II & 2.0 & 16 & 50 & 8.0 & 2.0 & 13 & 50 & 8.0 \\
\hline Ca II(HVF) & 25 & 28 & 50 & 4.0 & 6.0 & 26 & 50 & 4.0 \\
\hline Fe II(HVF) & 0.5 & 28 & 50 & 2.0 & 0.05 & 23 & 50 & 2.0 \\
\hline Fe III & 0.5 & 16 & 50 & 2.0 & 0.5 & 16 & 50 & 2.0 \\
\hline
\end{tabular}

Notes. $\tau$ : reference line optical depth, $v_{\min }$ : minimum velocity, $v_{\max }$ : maximum velocity, $v_{e}: e$-folding velocity of optical depth profile. All velocities are in units of $10^{3} \mathrm{~km} \mathrm{~s}^{-1}$. Both models assume $T_{\text {eff }}=12,000 \mathrm{~K}$.

\section{COMPARISON TO HVF IN OTHER SNe Ia}

Figure 11 shows the development of Si II $\lambda 6355$ absorption features by phase in six SNe Ia for which there are high-cadence spectroscopic observations that begin at least 12 days before $B$-max. ${ }^{15}$ We note that none of these SNe Ia are rapid decliners; their $\Delta m_{15}(B)$ values (in SN order of discovery) are 1.02, 1.05, $0.90,1.11,0.86$, and 0.80 .

The spectra obtained before -10 days provide strong evidence for detached $\mathrm{HVF}$ regions from both $\mathrm{Ca}$ II and $\mathrm{Si}$ II in five of the six SN. With one exception, the earliest spectra require separate HVF and PVF components to fit the line profiles. Only SN 2011fe at -16 days has a broad absorption feature that can be fit with a single Gaussian.

The line profiles for every spectrum in Figure 11 can be correlated with a profile from one of the phases in the spectral sequence of SN 2009ig. In some cases, the phase must be offset slightly to establish the best match. For example, the sequence of line profiles from -10 days to -5 days in SN 2005cf is nearly identical to the -12 days to -7 days profiles in SN 2009ig. The features of SN $2003 \mathrm{du}$ from -12 days to -8 days are similar to the features of SN 2009ig at the same phases, but the HVF diminish more rapidly in SN 2003du.

The most extreme phase offset relative to SN 2009ig is found in SN 2011fe, for which the sequence from -16 days to -10 days corresponds to SN 2009ig from -12 days to -6 days. Although the first spectrum of SN $2011 \mathrm{fe}$ was obtained earlier than the first spectrum of SN 2009ig with respect to $B$-max, the spectrum of SN $2011 \mathrm{fe}$ corresponds to a phase in the sequence that is after the detached HVF are observed. There is only a It is possible that even earlier observations of SN $2011 \mathrm{fe}$ could have revealed HVF.

Previous authors have studied the eccentricities of the Si II $\lambda 6355$ line profile in SNe Ia without directly detecting HVF. The presence of a HVF component from Si II $\lambda 6355$ is inferred from unusual line profiles that are wide, square, or triangular. None

\footnotetext{
15 Some of the data in Figure 11 were obtained through the WISeREP data archive (Yaron \& Gal-Yam 2012). The original published sources of the spectra are SN 2003du (Stanishev et al. 2007; Blondin et al. 2012), SN 2005cf (Garavini et al. 2007; Wang et al. 2009b), SN 2011fe (Nugent et al. 2011; Pereira et al. 2013) (SNFactory), and SN 2012fr (Childress et al. 2013b). The SN 2012cg data are previously unpublished FAST spectra from the CfA archive.
}

of the spectra available before SN 2009ig display separate and distinct HVF Si II features such as found at -14 and -13 days in SN 2009ig and from - 14 days to -11 days in SN $2012 \mathrm{fr}$.

Garavini et al. (2007) use SYNOW to model early-time spectra of eight $\mathrm{SNe}$ Ia. They show that fitting the Si II $\lambda 6355$ line profile requires a "detached" Si II region at 20,000-22,000 $\mathrm{km} \mathrm{s}^{-1}$ for seven of the eight SNe Ia. The other SNe in their sample requires a detached region at $24,500 \mathrm{~km} \mathrm{~s}^{-1}$.

Wang et al. (2009b) compare the early spectra of six SNe Ia and report that each of the Si II $\lambda 6355$ line profiles are well fit by a double-Gaussian function with separate central wavelengths. They show that two separate absorption features can be combined to produce flat-bottomed and triangular line profiles.

Figure 11 shows that for different $\mathrm{SNe}$ Ia the characteristic velocities of the HVF and PVF regions can vary by up to $3000 \mathrm{~km} \mathrm{~s}^{-1}$, but in all cases a gap of at least $5000 \mathrm{~km} \mathrm{~s}^{-1}$ is maintained between the HVF and PVF. Garavini et al. (2007), Stanishev et al. (2007), Wang et al. (2009b), and Childress et al. (2013b) describe the separation between HVF and PVF components to be about $8000 \mathrm{~km} \mathrm{~s}^{-1}$, the same value as the velocity gap in SN 2009ig.

The correlation of line profiles from pre-maximum spectra of other SNe Ia to the spectral sequence of SN 2009ig confirms a suggestion made by Stanishev et al. (2007) that the "peculiar" profiles of Si II $\lambda 6355$ in early-time spectra of SN Ia can be explained as part of a common evolutionary sequence that includes separate HVF and PVF components. Stanishev et al. (2007) also propose that the strength of the HVF components from Si II and Ca II lines is correlated in SNe Ia. Thus, if one of these lines produces strong HVF, then the other will also have a strong HVF component. The spectra of SN 2009ig confirm this relationship, and they also demonstrate that the presence of strong HVF from Si II and Ca II may be an indicator for HVF of Si III, S II, and Fe II.

The agreement between the SN 2009ig results and these well-studied SN Ia demonstrates that the behavior of HVF line forming regions in spectra of SN 2009ig is not unique, and in fact is quite common. The papers discussed in this section identify fifteen $\mathrm{SNe}$ Ia with spectra obtained at -10 days or earlier. All fifteen SN display HVF Si II at velocities between 17,000 and $22,000 \mathrm{~km} \mathrm{~s}^{-1}$, and all but SN $2011 \mathrm{fe}$ show evidence for separate HVF and PVF absorption components.

\section{POSSIBLE SOURCES OF HVF}

The observations of detached HVF in SN 2009ig suggest the presence of a region at $20,000-23,000 \mathrm{~km} \mathrm{~s}^{-1}$ in the velocity space of the SN with a localized enhancement of element abundance or density, or both. The proposed line forming region would be the source of HVF for Si II, S II, and Fe II from -12 days to -5 days.

This simple model is complicated by the fact that HVF are initially detected at a range of even higher velocities. In addition, the HVF velocities of Si II, Ca II, and Fe II decline at different rates. This suggests that the lines may sample different density structures. Although the velocity ranges overlap, the HVF of each line are not even detected in the same region. Figure 8 shows that at -14 days, Si II $\lambda 6355$ has the narrowest detached HVF with a velocity range from 10,000 to $30,000 \mathrm{~km} \mathrm{~s}^{-1}$. At the same phase, the HVF of CaII and Fe II HVF extend from 10,000 to $50,000 \mathrm{~km} \mathrm{~s}^{-1}$, but they are offset from each other. 


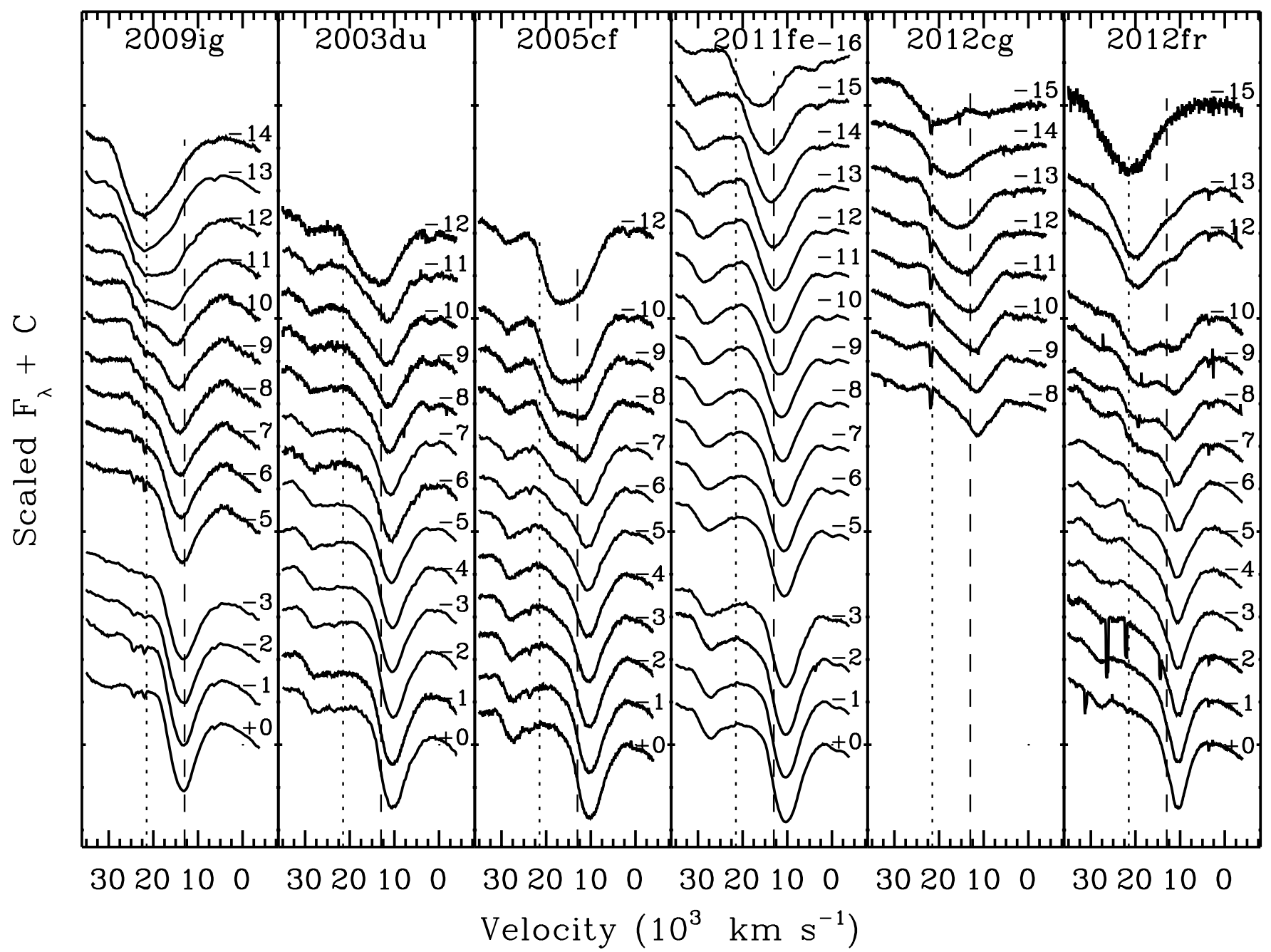

Figure 11. Si II $\lambda 6355$ absorption feature by phase in six SNe Ia, with the first observation at least 12 days before $B$-max. The earliest data clearly require separate HVF and PVF components to fit the line profiles, except for SN 2011fe which forms a single broad absorption feature. The HVF and PVF components appear together for a few days. After -6 days only the PVF is detected, and all spectra show similar line profiles with small differences between the SN in velocity space. The sequence of line profiles from -10 days to -5 days in SN $2005 \mathrm{cf}$ is nearly identical to the -12 days to -7 days profiles from SN $2009 \mathrm{ig}$. All of the spectra in this figure can be correlated with a line profile in the sequence of SN 2009ig. The dotted line at 21,500 km s $\mathrm{km}^{-1}$ and the dashed line at $13,000 \mathrm{~km} \mathrm{~s}^{-1}$ are the same in each panel.

Ca II absorptions are exceptionally broad in velocity space, with absorption minima that are several thousand $\mathrm{km} \mathrm{s}^{-1}$ higher than the HVF components of other lines. Due to the very low excitation potentials of the Ca II lines $(\leqslant 1.7 \mathrm{eV})$, they are capable of producing detectable absorptions at much lower abundances than most other lines found in SNe Ia. Fe II lines also have low excitation potentials $(\leqslant 2.9 \mathrm{eV})$. In SN 2009ig, the HVF from Fe II are nearly as wide and deep as HVF of Ca II, with velocities between $\mathrm{Ca}$ II and $\mathrm{Si}$ II.

The simplest explanation for both the HVF $\mathrm{Ca}$ and $\mathrm{Fe}$ observations is that primordial abundances of these elements are sufficient to produce the observed absorptions. The line profiles can be produced by light from the SN passing through a line forming region that extends from about 10,000 to $50,000 \mathrm{~km} \mathrm{~s}^{-1}$ in velocity space. The observed minima for these features would move to lower velocities with time, as expansion of the SN reduces opacity for HVFs more rapidly than it does at lower velocities. As the column depth is reduced, the observed minima become nearly constant at velocities near to the inner edge of the line forming region.

If the HVF of CaII and $\mathrm{Fe}$ II are formed in regions of unburned material from the progenitor, it is still a challenge to explain the simultaneous presence of detached HVF for Si II $\lambda 6355(8.1 \mathrm{eV})$ and $\mathrm{S}$ II $(13.7 \mathrm{eV})$. An explanation is also required for the fact that the HVF velocities for all lines, as measured at the absorption minima, eventually converge near $21,500 \mathrm{~km} \mathrm{~s}^{-1}$. Since Figure 8 shows that the line forming materials are distributed through a wide range of velocities, there must be some motivation for the line opacities of Si II, $\mathrm{Si}$ III, S II, Ca II, and Fe II to have approximately equal values at the same phases and in a relatively narrow velocity range.

Attempts have been made to explain HVF without invoking a separate line forming region. The flat-bottomed and triangular shapes observed in the Si II $\lambda 6355$ line profile at early times receive particular attention. None of the physical models used to generate unusual line profiles survive comparisons with early observations of multiple SN Ia. HVF from Ca II have also been attributed to opacity effects from recombination, but the time scale of recombination is a day or two and HVF from Ca II are observed from 8 to 15 days in individual SNe Ia.

Explosion models for SNe Ia make predictions for structures that range from spherical to highly asymmetric. The resulting chemical distributions may be simply stratified by atomic mass, violently mixed between regions of burned material, or contain 
bubbles of material moving between burning regions. Model results can be tuned to produce specific layering compositions and velocity distributions that may mimic HVF of specific atoms at specific locations. We are not aware of any explosion models that predict the relative uniformity across a range of absorbing materials that is observed for HVF in SN 2009ig.

One way to increase the local density in HVF regions is by interaction between the expanding ejecta and nearby circumstellar material (CSM; Gerardy et al. 2004). The shock front sweeps up CSM and a shell is "locked in" after the shock moves on. The shell will continue in homologous expansion with the rest of the SN ejecta. This model satisfies the constraint that the HVF line forming region exist in a narrow, unchanging velocity window and explains the asymmetric line profiles of the Si II $\lambda 6355$ HVF.

Three of the recent models have been able to produce variable density structures: symmetric ignitions that generate strong mixing (Maeda et al. 2010), gravitationally confined detonations with burning regions on the surface of the WD (Meakin et al. 2009), and double-detonation models that produce an HVF burning region with a density gap between it and the center of the SN (Shen et al. 2013).

\section{SUMMARY AND CONCLUSIONS}

We present a time series of spectra of SN 2009ig obtained between -14.5 days and +12.5 days with respect to the time of $B$-max. The two earliest observations in our sample ( -14.5 days and -13.5 days) are the first spectra of an SN Ia to resolve the HVF component of Si II $\lambda 6355$ as a distinctly separate absorption feature. HVF are identified in the spectra from Si II, Si III, S II, Ca II, and Fe II, and we show that the line profiles require a detached $\mathrm{HVF}$ line forming region.

Simultaneous detections are made for HVF and PVF of the same lines from -12 days to -5 days. Velocity measurements demonstrate that the HVF and PVF line forming regions are separated by $\sim 8000 \mathrm{~km} \mathrm{~s}^{-1}$ as long as HVF are detected. Using a mean velocity of $21,500 \mathrm{~km} \mathrm{~s}^{-1}$ for the HVF layer and $13,000 \mathrm{~km} \mathrm{~s}^{-1}$ for the PVF layer, we show that $R_{\mathrm{HVF}} \approx$ 1.6 $R_{\mathrm{PVF}}$.

The initial HVF velocities and the velocity decline rates are different for each line. The fact that the HVF velocities eventually converge suggests the presence of a line forming region in SN 20019ig at velocities between 20,000 and 23,000 $\mathrm{km} \mathrm{s}^{-1}$. A comparison of the HVF from Si II, Ca II, and Fe II by phase in velocity space shows that HVF from different lines are formed in overlapping but not identical regions. HVF of $\mathrm{Ca}$ II and $\mathrm{Fe}$ II have strong features that range from 10,000 to $50,000 \mathrm{~km} \mathrm{~s}^{-1}$ in the earliest spectra, but the locations of their absorption minima differ by $\sim 7000 \mathrm{~km} \mathrm{~s}^{-1}$. At the same phases, Si II HVF are confined to a much narrower region that extends from 10,000 to only $30,000 \mathrm{~km} \mathrm{~s}^{-1}$. We explore the physical conditions that can produce detached HVF, and find that it is challenging for any model to reproduce the observations of SN 2009ig.

The spectra of SN 2009ig form a complete map of the transition from the earliest observations of detached HVF to features with contributions from both HVF and PVF, and finally to phases when all of the absorptions are PVF. HVF of both $\mathrm{Ca}$ II and $\mathrm{Si}$ II are frequent characteristics of SNe Ia observed before -10 days, and that allows us to compare our results to observations of several other SNe Ia. All of the line profiles found in early-time spectra of SNe Ia can easily be correlated with individual profiles that are part of the development sequence defined by SN 2009ig. We interpret this as evidence for a common evolutionary sequence in $\mathrm{SNe}$ Ia that requires both $\mathrm{HVF}$ and PVF line forming regions.

The authors wish to thank the Chairmen of the TACs from the University of Texas and Penn State University for providing Director's discretionary time for the HET/LRS observations. G.H.M. thanks Nick Suntzeff and Rob Robinson for insightful comments. G.H.M. also thanks Mark Phillips for providing perspective, as well as Michael Childress for helpful discussions and for sharing an advance copy of his paper on SN 2012fr. We thank Mark Sullivan, Isobel Hook, Peter Nugent, Andy Howell, and Bill Vacca for sharing their own data on line profiles in young SNe Ia. The authors make frequent use of David Bishop's excellent Web site listing recent supernovae and valuable references associated with them: http://www.rochesterastronomy.org/snimages/. J.V. is supported by Hungarian OTKA Grants K-76816 and NN-107637, NSF grant AST-0707769, and Texas Advanced Research Project ARP-009. The research of J.C.W. in supported in part by NSF grant AST-1109801. The CfA Supernova Program is supported by NSF grant AST-1211196 to the Harvard College Observatory. X. Wang is supported by the Natural Science Foundation of China (NSFC 11178003, 11073013), the China-973 Program 2009CB824800, and NSF grant AST-0708873 (through L. Wang). E.Y.H. is supported by NSF grant AST-1008343. A.V.F. is grateful for financial assistance from NSF grant AST-1211916, the TABASGO Foundation, and the Christopher R. Redlich Fund. We greatly appreciate the valuable assistance provided by staff members at the observatories where SN 2009ig was observed.

Facilities: FLWO:1.5m (FAST), HET (LRS), Keck:I (LRIS), MMT (Blue Channel), Shane (Kast), Swift (UVOT; UV grism)

\section{REFERENCES}

Benetti, S., Cappellaro, E., Mazzali, P. A., et al. 2005, ApJ, 623, 1011 Blondin, S., Matheson, T., Kirshner, R. P., et al. 2012, AJ, 143, 126 Branch, D., Baron, E., Hall, N., Melakayil, M., \& Parrent, J. 2005, PASP, 117,545

Branch, D., Dang, L. C., \& Baron, E. 2009, PASP, 121, 238

Branch, D., Garnavich, P., Matheson, T., et al. 2003, AJ, 126, 1489

Branch, D., Jeffery, D. J., Parrent, J., et al. 2008, PASP, 120, 135

Branch, D., Troxel, M. A., Jeffery, David J., et al. 2007, PASP, 119, 709

Childress, M. J., Filippenko, A. V., Ganeshalingam, M., \& Schmidt, B. P. 2013a, MNRAS, submitted (arXiv:1307.0563)

Childress, M. J., Scalzo, R. A., Sim, S. A., et al. 2013b, ApJ, 770, 29

Filippenko, A. V. 1982, PASP, 94, 715

Filippenko, A. V., Li, W. D., Treffers, R. R., \& Modjaz, M. 2001, in ASP Conf. Ser. 246, Small-Telescope Astronomy on Global Scales, ed. B. Paczyński, W. P. Chen, \& C. Lemme (San Francisco, CA: ASP), 121

Fisher, A., Branch, D., Nugent, P., \& Baron, E. 1997, ApJ, 481, 89 Foley, R. J. 2013, MNRAS

Foley, R. J., Challis, P. J., Filippenko, A. V., et al. 2012, ApJ, 744, 38

Foley, R. J., Papenkova, M. S., Swift, B. J., et al. 2003, PASP, 115, 1220

Foley, R. J., Sanders, N. E., \& Kirshner, R. P. 2011, ApJ, 742, 89

Garavini, G., Nobili, S., Taubenberger, S., et al. 2007, A\&A, 471, 527

Gehrels, N., Chincarini, G., Giommi, P., et al. 2004, ApJ, 611, 1005

Gerardy, C. L., Höflich, P., Fesen, R. A., et al. 2004, ApJ, 607, 391

Hatano, K., Branch, D., Fisher, A., Baron, E., \& Filippenko, A. V. 1999, ApJ, 525,881

Hill, G. J., Nicklas, H. E., MacQueen, P. J., et al. 1998, Proc. SPIE, 3355, 375

Horne, K. 1986, PASP, 98, 609

Hoyle, P., \& Fowler, W. A. 1960, ApJ, 132, 565

Jha, S., Riess, A. G., \& Kirshner, R. P. 2007, ApJ, 659, 122

Kasen, D., Nugent, P., Wang, L., et al. 2003, ApJ, 593, 788

Kleiser, I., Cenko, S. B., Li, W., \& Filippenko, A. V. 2009, CBET, 1918, 1

Maeda, K., Röpke, F. K., Fink, M., et al. 2010, ApJ, 712, 624

Marion, G. H., Höflich, P., Gerardy, C. L., et al. 2009, AJ, 138, 727

Matheson, T., Filippenko, A. V., Ho, L. C., Barth, A. J., \& Leonard, D. C. 2000, AJ, 120, 1499 
Mattila, S., Lundqvist, P., Sollerman, J., et al. 2005, A\&A, 443, 649 Mazzali, P. A., Benetti, S., Altavilla, G., et al. 2005a, ApJL, 623, L37 Meakin, C. A., Seitenzahl, I., Townsley, D., et al. 2009, ApJ, 693, 1188 Miller, J. S., \& Stone, R. P. S. 1993, Lick Obs. Tech. Rep. 66 (Santa Cruz, CZ: Lick Obs.)

Navasardyan, H., Cappellaro, E., \& Benetti, S. 2009, CBET, 1918, 1 Nugent, P. E., Sullivan, M., Cenko, S. B., et al. 2011, Natur, 480, 344 Oke, J. B., Cohen, J. G., Carr, M., et al. 1995, PASP, 107, 375

Parrent, J. T., Thomas, R. C., Fesen, R. A., et al. 2011, ApJ, 732, 30

Patat, F., Baade, D., Höflich, P., et al. 2009, A\&A, 508, 229

Pereira, R., et al. 2013, A\&A, 554, 27

Quimby, R., Höflich, P., Kannappan, S. J., et al. 2006, ApJ, 636, 400

Ramsey, L. W., et al. 1998, Proc. SPIE, 3352, 34

Roming, P. W. A., Kennedy, T. E., Mason, K. O., et al. 2005, SSRv, 120, 95

Schlafly, E. F., \& Finkbeiner, D. P. 2011, ApJ, 737, 103
Schmidt, G. D., Weymann, R. J., \& Foltz, C. B. 1989, PASP, 101, 713

Shen, K. J., Guillochon, J., \& Foley, R. J. 2013, ApJL, 770, L35

Silverman, J. M., Kong, J. J., \& Filippenko, A. V. 2012, MNRAS, 25, 1819

Stanishev, V., Goobar, A., Benetti, S., et al. 2007, A\&A, 469, 645

Tanaka, M., Mazzali, P. A., Benetti, S., et al. 2008, ApJ, 677, 448

Tanaka, M., Mazzali, P. A., Maeda, K., \& Nomoto, K. 2006, ApJ, 645, 470

Thomas, R. C., Branch, D., Baron, E., et al. 2004, ApJ, 601, 1019

Wade, R. A., \& Horne, K. 1988, ApJ, 324, 411

Wang, L., Baade, D., Höflich, P., et al. 2003, ApJ, 591, 1110

Wang, L., Baade, D., Höflich, P., et al. 2006, ApJ, 653, 490

Wang, X., Filippenko, A. V., Ganeshalingam, M., et al. 2009a, ApJL, 699, L139

Wang, X., Li, W., Filippenko, A. V., et al. 2008, ApJ, 675, 626

Wang, X., Li, W., Filippenko, A. V., et al. 2009b, ApJ, 697, 380

Yaron, O., \& Gal-Yam, A. 2012, PASP, 124, 668 